\title{
Mapping the kinematical regimes of semi-inclusive deep inelastic scattering
}

\section{Boglione, ${ }^{a}$ A. Dotson, ${ }^{b}$ L. Gamberg, ${ }^{c}$ S. Gordon, ${ }^{d}$ J.O. Gonzalez-Hernandez, ${ }^{a}$}

A. Prokudin, ${ }^{e, c}$ T.C. Rogers ${ }^{e, d}$ and N. Sato ${ }^{e}$

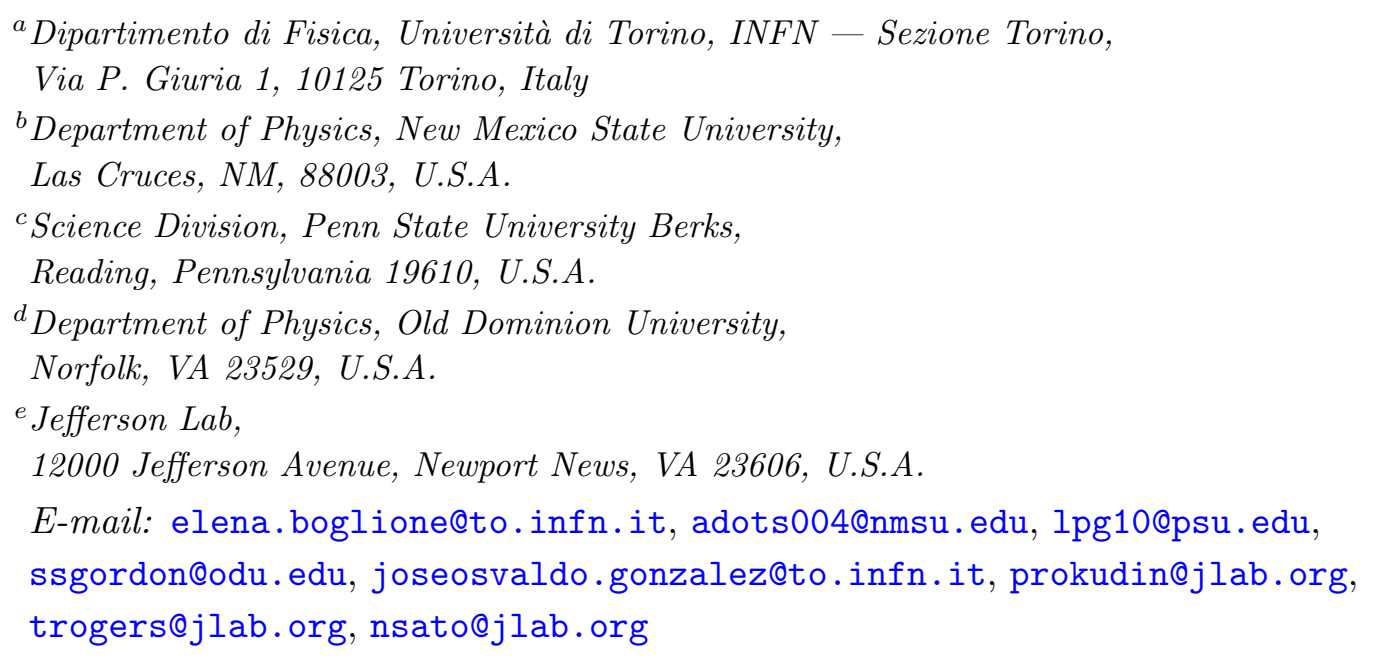

ABSTRACT: We construct a language for identifying kinematical regions of transversely differential semi-inclusive deep inelastic scattering (SIDIS) cross sections with particular underlying partonic pictures, especially in the regions of moderate to low $Q$ where sensitivity to kinematical effects becomes non-trivial. These partonic pictures map to power law expansions whose leading contributions ultimately lead to well-known QCD factorization theorems. In order to establish the consistency of a particular observable in SIDIS process with an estimate of the appropriate underlying partonic picture, we introduce new quantitative criteria expressed in terms of various ratios of partonic and hadronic momentum degrees of freedom. We propose how to use these criteria in phenomenology and provide a web tool which allows visualization of these ratios for any chosen kinematic configuration.

KeYwords: Deep Inelastic Scattering (Phenomenology), QCD Phenomenology

ARXiv EPrint: 1904.12882 


\section{Contents}

1 Introduction 1

2 The SIDIS process 3

2.1 Reference frames 5

2.1.1 Photon frame 5

2.1.2 Hadron frame 6

2.1.3 Breit frame 6

2.2 The SIDIS cross section 8

$\begin{array}{lll}3 & \text { Kinematical approximations } & 10\end{array}$

4 Mapping SIDIS kinematics and partonic subprocesses $\quad 14$

$\begin{array}{ll}4.1 \text { TMD current region } & 17\end{array}$

$\begin{array}{llr}4.2 \text { Hard transverse momentum } & 18\end{array}$

$\begin{array}{ll}4.3 \text { Target and soft regions } & 19\end{array}$

$\begin{array}{lll}5 & \text { Numerical examples } & 21\end{array}$

6 Conclusions $\quad 26$

$\begin{array}{lr}\text { A Light-cone variables } & 28\end{array}$

$\begin{array}{ll}\text { B Lorentz transformations } & 28\end{array}$

$\begin{array}{ll}\text { C Cross sections and structure functions } & 29\end{array}$

$\begin{array}{ll}\text { D Sum rule } & 32\end{array}$

$\begin{array}{lll}\text { E Rapidity } & 33\end{array}$

\section{Introduction}

Deep inelastic scattering (DIS), and especially semi-inclusive deep inelastic scattering (SIDIS) are headlining processes in most programs to the study of partonic (quark and gluon) degrees of freedom. It is a cornerstone process of, for example, the Jefferson Lab $12 \mathrm{GeV}$ program to study partonic structure in hadrons, and is one of the important processes for study in a future Electron-Ion Collider (EIC) [1-6]. Interest in SIDIS arises from a variety of considerations. Well-established collinear factorization theorems for SIDIS provide access to the flavor dependence of standard parton distribution functions (PDFs) and fragmentation functions (FFs) in the so-called current fragmentation region. In the 
target fragmentation region, different kinds of objects, called fracture functions $[7,8]$, are involved and these are sensitive to still other novel QCD phenomena. Beyond collinear factorization, transversely differential SIDIS at low transverse momentum is sensitive to the properties of transverse momentum dependent (TMD) PDFs and FFs.

Many DIS experiments are performed at moderate-to-low $Q$ (roughly $1-3 \mathrm{GeV}$ ), ${ }^{1}$ where non-perturbative effects are significant and it is reasonable to expect sensitivity to intrinsic properties of hadrons. The moderate-to-low $Q$ region has some obvious advantages in the mission to refine the current view of hadron structure. If all energies and hard scales are extremely large, then asymptotic freedom means that pictures of partonic interactions rooted in perturbation theory can usually be applied confidently and with very high accuracy and precision. But, with the large relative fraction of the hard process contributions and perturbatively produced radiation involved, it becomes less clear to what extent observables are truly sensitive to the intrinsic properties of the actual hadron constituents. This further points to moderate-to-low $Q$ measurements as ideal sources of information about partonic hadron structure. However, there are also unique challenges to interpreting moderate-to-low $Q$ cross sections, particularly for less inclusive versions of DIS like SIDIS. With lower hard scales, access to intrinsic effects of constituents may be more direct, but this also comes with less confidence in the reliability and accuracy of perturbative and/or parton-based descriptions. Moreover, the average final state hadron multiplicity in such measurements is typically very low in the valence region of Bjorken- $x_{\mathrm{Bj}}$. In long term efforts to establish intrinsic properties for partons, the trade-off in advantages at large and small $Q$ needs to be confronted systematically, and such that knowledge of one complements the other.

Sophisticated theoretical frameworks, usually involving some form of QCD factorization and perturbation theory [9-11] have long existed for describing specific underlying physical mechanisms in many highly differential processes over many regions, including in SIDIS, in terms of partonic degrees of freedom. However, they always assume specific kinematical limiting cases, e.g. very large or very small transverse momentum, or very large or very small rapidity. The interface between different physical regimes remains somewhat unclear in practice, and most especially when the hard scales involved are not large. Estimating the kinematical boundaries of any specific QCD approach or approximation beyond very rough orders of magnitude is difficult and subtle. Within the current experimental and phenomenological knowledge it requires at least some model assumptions, e.g. about the role of parton virtuality and/or the onset of various non-perturbative or hadronic mechanisms generally. Monte Carlo simulations can help, but these also involve physical assumptions whose impact needs to be understood systematically. Future phenomenological and experimental efforts will hopefully clarify the location of region boundaries, and discriminate between competing hypotheses.

The aim of the present article is to discuss how such questions can be posed in a systematic way. To this end, we will refrain from discussing specific theoretical frameworks

\footnotetext{
${ }^{1}$ By "moderate-to-low $Q$," we will mean SIDIS measurements with $Q$ roughly between $1 \mathrm{GeV}$ and $3 \mathrm{GeV}$ and Bjorken- $x_{\mathrm{Bj}}$ not too far below the valence region. This includes, in particular, JLab $6 \mathrm{GeV}$ and $12 \mathrm{GeV}$ SIDIS cross section measurements $[1,4,6]$.
} 
and instead enumerate the steps needed to map any given set of assumptions concerning exact intrinsic partonic/constituent properties to a corresponding kinematical region of $x_{\mathrm{Bj}}, Q, z_{\mathrm{h}}$, and transverse momentum in a cross section. The goal is to construct basic elements needed for an interpretation strategy applicable with any model of underlying non-perturbative dynamics for exact parton momentum, independent even of assumptions about factorization. Our final result is a set of novel region indicators ${ }^{2}$ expressed in terms of partonic and hadronic degrees of freedom that probe the proximity of any given kinematical configuration to a particular conventional partonic region of SIDIS; such as current region, target fragmentation region, or soft region.

In the first part of the paper we provide an overview of the SIDIS process, in some cases translating past results into an updated language, motivated by current research efforts. ${ }^{3}$ section 2 treats the kinematics, notation and setup, and explains the kinematical characterization of final state hadron momentum. In section 2 we also introduce the cross section and its exact decomposition in terms of structure functions, for which we extend the sum rule derived originally in ref. [20].

In the second part of the paper, section 3, we discuss the standard approximations used to characterize SIDIS processes, and focusing on those of purely kinematical origin we propose a simple test of the quality of the commonly used massless-hadron approximation (MHA) [23]. In section 4 we explain the characterization of partonic kinematics and establish a language to connect such pictures to specific observable kinematical regions, with a focus on the current fragmentation section 4.1 and large transverse momentum regimes section 4.2. In section 4.3 we discuss the target and soft regions. In section 5, we provide some examples of the region characterization in terms of the $R$ ratios, within typical experimental kinematics. Finally, we make concluding remarks in section 6 .

\section{The SIDIS process}

We consider the process:

$$
\operatorname{lepton}(l)+\operatorname{proton}(P) \rightarrow \operatorname{lepton}\left(l^{\prime}\right)+\operatorname{Hadron}\left(P_{\mathrm{B}}\right)+X .
$$

The final state hadron has type $B$. The " $X$ " is an instruction to sum over all unobserved particles including other $B$ type hadrons. A sketch is shown in figure $1 .^{4}$ The proton has momentum $P$, the virtual photon has momentum $q$, the produced hadron has momentum $P_{\mathrm{B}}$, and the incoming and scattered leptons have momenta $l$ and $l^{\prime}$ respectively. The mass of the target hadron is $M$ and the mass of the produced hadron is $M_{\mathrm{B}}$.

\footnotetext{
${ }^{2}$ We provide a convenient web interface which can be found at ref. [12].

${ }^{3}$ For general introductions to SIDIS in pQCD see, for example, refs. [13-18]. See refs. [19-22] for review of the basics of SIDIS that includes a full catalogue of spin and azimuthal dependencies. For general treatments of SIDIS in the context of fracture functions and target fragmentation, see ref. [8]. Also see chapters $12-13$ of ref. [11], which influences much of the language and notation of this article.

${ }^{4}$ In this figure we followed the so-called Trento conventions [24].
} 


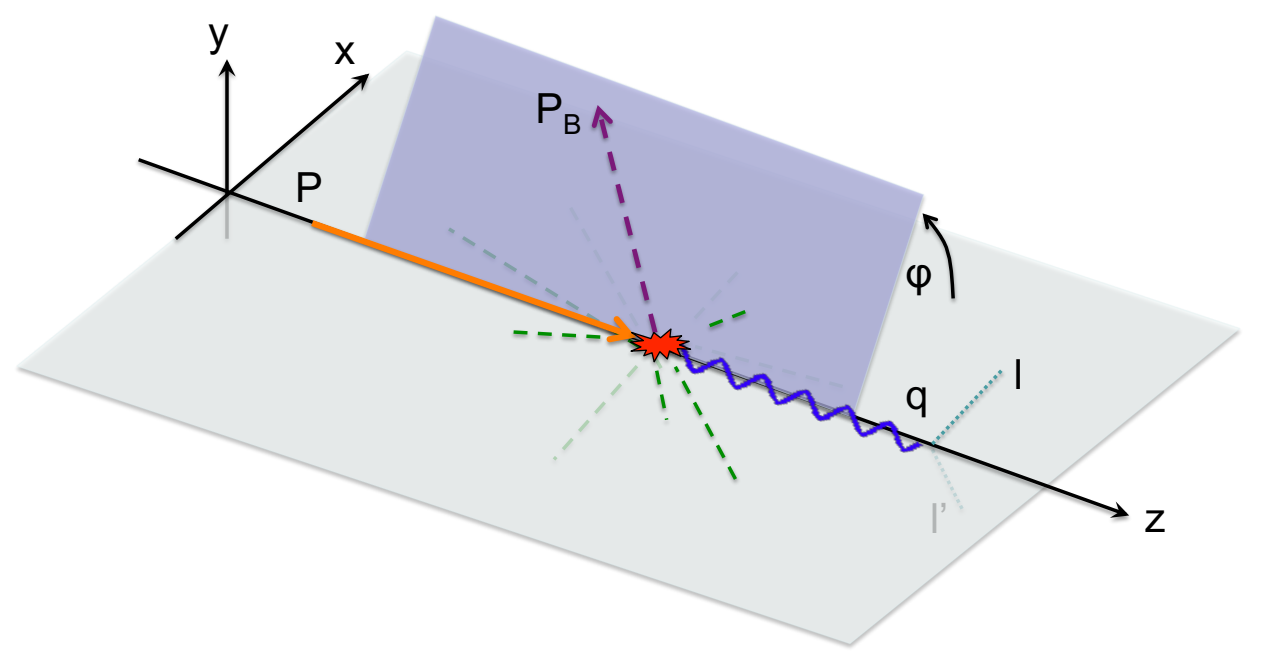

Figure 1. The diagram of a SIDIS event in a photon frame. The hadron plane is shown in purple. The dashed green lines represent unobserved particles.

Observables, like cross sections or structure functions, are conventionally parameterized by a combination of the following kinematical variables:

$$
\begin{aligned}
Q^{2} & =-q^{2}=-\left(l-l^{\prime}\right)^{2}, \quad x_{\mathrm{Bj}}=\frac{Q^{2}}{2 P \cdot q}, \\
x_{\mathrm{N}} & =-\frac{q^{+}}{P^{+}}=\frac{2 x_{\mathrm{Bj}}}{1+\sqrt{1+\frac{4 x_{\mathrm{Bj}}^{2} M^{2}}{Q^{2}}}}, \\
y & =\frac{P \cdot q}{P \cdot l}, \quad z_{\mathrm{h}}=\frac{P \cdot P_{\mathrm{B}}}{P \cdot q}=2 x_{\mathrm{Bj}} \frac{P \cdot P_{\mathrm{B}}}{Q^{2}}, \quad z_{\mathrm{N}}=\frac{P_{\mathrm{B}}^{-}}{q^{-}}, \quad s=(l+P)^{2} . \\
W_{\mathrm{tot}}^{2} & =(q+P)^{2}, \quad W_{\mathrm{SIDIS}}^{2}=\left(q+P-P_{\mathrm{B}}\right)^{2}, \quad s \quad l
\end{aligned}
$$

In the expressions of the light-cone ratios that define $x_{\mathrm{N}}$ and $z_{\mathrm{N}}$, momentum components $q^{ \pm}, P^{+}$and $P_{\mathrm{B}}^{-}$are defined in a photon frame, figure 1 , where the incoming proton is in the positive $z$-direction with zero transverse momentum and the virtual photon is the negative $z$-direction with no transverse momentum.

Since boosts along the $z$-axis do not affect light-cone ratios, the exact photon frame does not matter. The variable $x_{\mathrm{N}}$ is the kinematical variable usually called Nachtmann- $x$. It is often labeled by a $\xi$ in the literature, but for us $\xi$ will label a partonic momentum fraction, so we use $x_{\mathrm{N}}$ instead; with the subscripts on $x_{\mathrm{N}}$ and $x_{\mathrm{Bj}}$ distinguishing between Bjorken and Nachtmann $x$-variables. For descriptions of fragmentation, the light-cone fraction $z_{\mathrm{N}}$ is the analogue of $x_{\mathrm{N}}$, and the $\mathrm{N}$ subscript is meant to emphasize this analogy. Our $x_{\mathrm{Bj}}$, $z_{\mathrm{h}}$ and $P_{\mathrm{B}}$ correspond, respectively, to $x, z$ and $P_{h}$ from ref. [20]. Our $z_{\mathrm{N}}$ corresponds to $\zeta_{h}$ of ref. [25]. A variable

$$
x_{\mathrm{h}}=\frac{q \cdot P_{\mathrm{B}}}{P \cdot q}
$$

is useful if the target fragmentation region is being described. 
The deep inelastic limit is $m / Q \rightarrow \infty$ with fixed $x_{\mathrm{N}}$ and $z_{\mathrm{N}}$. The " $m$ " symbol will always represent a generic mass scale in this paper, considered to be very small relative to $Q$, such as a small hadron mass or $\Lambda_{\mathrm{QCD}}$. The kinematical variables obey

$$
Q^{2}=x_{\mathrm{Bj}} y\left(s-M^{2}-m_{l}^{2}\right) \approx x_{\mathrm{Bj}} y s .
$$

The " $\approx$ " symbol will always mean "dropping $m / Q$ power-suppressed corrections" with $x_{\mathrm{N}}$ and $z_{\mathrm{N}}$ fixed.

Next we treat the final state B momentum in terms of the light-cone momentum fraction $z_{\mathrm{N}}$, eq. (2.3), variable and relate it to the transverse momentum of the photon. In order to do this it is important to be able to express the final state momentum in both the photon and hadron frames.

\section{$2.1 \quad$ Reference frames}

To establish our notation we will use the following representation of virtual photon and hadron momenta in photon and hadron reference frames in light-cone coordinates and rapidity. ${ }^{5}$

\subsubsection{Photon frame}

In a photon frame, the virtual photon and the initial proton both have zero transverse momentum, while the final state produced hadron has non-zero transverse momentum:

$$
\begin{aligned}
q_{\gamma} & =\left(-x_{\mathrm{N}} P_{\gamma}^{+}, \frac{Q^{2}}{2 x_{\mathrm{N}} P_{\gamma}^{+}}, \mathbf{0}_{\mathrm{T}}\right), \\
P_{\gamma} & =\left(P_{\gamma}^{+}, \frac{M^{2}}{2 P_{\gamma}^{+}}, \mathbf{0}_{\mathrm{T}}\right), \\
P_{\mathrm{B}, \gamma} & =\left(\frac{\mathbf{P}_{\mathrm{B}, \gamma, \mathrm{T}}^{2}+M_{\mathrm{B}}^{2}}{2 P_{\mathrm{B}, \gamma}^{-}}, P_{\mathrm{B}, \gamma}^{-}, \mathbf{P}_{\mathrm{B}, \gamma, \mathrm{T}}\right) .
\end{aligned}
$$

Note that eq. (2.7) fixes $x_{\mathrm{N}}$ to be defined as in eq. (2.2).

The $\gamma$ subscript signals the use of components in the photon frame, following the notation of eq. (A.7). In the photon frame

$$
P_{\mathrm{B}, \gamma}^{-}=\frac{z_{\mathrm{h}} Q^{2}}{4 x_{\mathrm{Bj}} P_{\gamma}^{+}}\left(1 \pm \sqrt{1-\frac{4 x_{\mathrm{Bj}}^{2} M^{2}\left(\mathbf{P}_{\mathrm{B}, \gamma, \mathrm{T}}^{2}+M_{\mathrm{B}}^{2}\right)}{z_{\mathrm{h}}^{2} Q^{4}}}\right) \approx \frac{z_{\mathrm{h}} Q^{2}}{2 x_{\mathrm{Bj}} P_{\gamma}^{+}},
$$

where the approximation symbol shows the limit of zero hadron masses for the solution, "+", corresponding to the current fragmentation region. Note that ratios of plus and minus components are independent of boosts in the $z$-direction, and so are the same in all photon frames.

\footnotetext{
${ }^{5}$ See appendix A and appendix E for conventions on light-cone coordinates and rapidity respectively.
} 


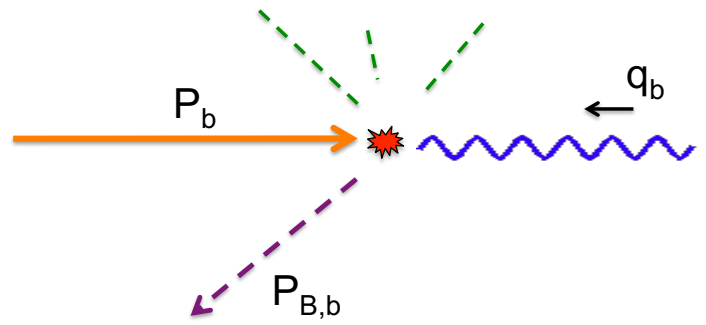

(a)

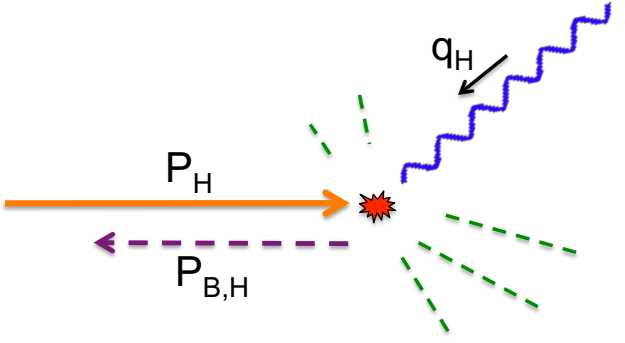

(b)

Figure 2. The configuration of the proton, photon, and outgoing hadron in (a) the Breit photon frame and (b) the hadron frame. The dashed green lines again represent unobserved particles.

\subsubsection{Hadron frame}

In the hadron frame, see figure 2(b), labeled by "H," the incoming hadron and final state hadron are exactly back-to-back (zero relative transverse momentum) while the virtual photon generally has non-zero transverse momentum. It is an especially useful frame for setting up factorization. (See [11, section13.15.5].) The components of the four-momenta are:

$$
\begin{aligned}
q_{\mathrm{H}} & =\left(q_{\mathrm{H}}^{+}, q_{\mathrm{H}}^{-}, \mathbf{q}_{\mathrm{H}, \mathrm{T}}\right), \\
P_{\mathrm{H}} & =\left(P_{\mathrm{H}}^{+}, \frac{M^{2}}{2 P_{\mathrm{H}}^{+}}, \mathbf{0}_{\mathrm{T}}\right), \\
P_{\mathrm{B}, \mathrm{H}} & =\left(\frac{M_{\mathrm{B}}^{2}}{2 P_{\mathrm{B}, \mathrm{H}}^{-}}, P_{\mathrm{B}, \mathrm{H}}^{-}, \mathbf{0}_{\mathrm{T}}\right) .
\end{aligned}
$$

For definiteness, we express the hadron frame such that the components of the incoming target momentum are exactly the same as in the Breit frame:

$$
\begin{aligned}
P_{\mathrm{H}}^{+} & =P_{\gamma}^{+}=\frac{Q}{\sqrt{2} x_{\mathrm{N}}}, \\
P_{\mathrm{H}}^{-} & =P_{\gamma}^{-}=\frac{x_{\mathrm{N}} M^{2}}{\sqrt{2} Q}, \\
\mathbf{P}_{\mathrm{H}, \mathrm{T}} & =\mathbf{0}_{\mathrm{T}} .
\end{aligned}
$$

\subsubsection{Breit frame}

A particular case of the photon frame is the Breit (Brick Wall) frame, see figure 2(a), where

$$
\begin{aligned}
q_{\mathrm{b}} & =\left(-\frac{Q}{\sqrt{2}}, \frac{Q}{\sqrt{2}}, \mathbf{0}_{\mathrm{T}}\right), \\
P_{\mathrm{b}} & =\left(\frac{Q}{x_{\mathrm{N}} \sqrt{2}}, \frac{x_{\mathrm{N}} M^{2}}{\sqrt{2} Q}, \mathbf{0}_{\mathrm{T}}\right)=\left(\frac{M}{\sqrt{2}} e^{y_{P, \mathrm{~b}}}, \frac{M}{\sqrt{2}} e^{-y_{P, \mathrm{~b}}}, \mathbf{0}_{\mathrm{T}}\right) .
\end{aligned}
$$

The small b indicates that components are in the Breit frame. This will be our default frame, so any four-momentum components without a subscript should be assumed to be in the Breit frame. 
Now we specialize to the Breit frame, where the exact final state hadron $P_{\mathrm{B}, \mathrm{b}}$ as measured in experiment is

$$
P_{\mathrm{B}, \mathrm{b}}=\left(\frac{M_{\mathrm{B}, \mathrm{T}}^{2}}{2 P_{\mathrm{B}, \mathrm{b}}^{-}}, P_{\mathrm{B}, \mathrm{b}}^{-}, \mathbf{P}_{\mathrm{B}, \mathrm{b}, \mathrm{T}}\right)=\left(\frac{M_{\mathrm{B}, \mathrm{T}}}{\sqrt{2}} e^{y_{\mathrm{B}, \mathrm{b}}}, \frac{M_{\mathrm{B}, \mathrm{T}}}{\sqrt{2}} e^{-y_{\mathrm{B}, \mathrm{b}}}, \mathbf{P}_{\mathrm{B}, \mathrm{b}, \mathrm{T}}\right),
$$

where $M_{\mathrm{B}, \mathrm{T}}=\sqrt{M_{\mathrm{B}}^{2}+\mathbf{P}_{\mathrm{B}, \mathrm{b}, \mathrm{T}}^{2}}$ is the transverse mass, $y_{\mathrm{B}, \mathrm{b}}$ is the produced hadron rapidity, and $P_{\mathrm{B}, \mathrm{b}}^{-}=z_{\mathrm{N}} q_{\mathrm{b}}^{-}=z_{\mathrm{N}} Q / \sqrt{2}$.

It is then convenient to express $P_{\mathrm{B}, \mathrm{b}}$ in the Breit frame in terms of $z_{\mathrm{N}}$, eq. (2.3), and a new variable $\mathbf{q}_{\mathrm{T}}$ as follows

$$
P_{\mathrm{B}, \mathrm{b}}=\left(\frac{M_{\mathrm{B}}^{2}+z_{\mathrm{N}}^{2} \mathbf{q}_{\mathrm{T}}^{2}}{\sqrt{2} z_{\mathrm{N}} Q}, \frac{z_{\mathrm{N}} Q}{\sqrt{2}},-z_{\mathrm{N}} \mathbf{q}_{\mathrm{T}}\right),
$$

where $\mathbf{q}_{\mathrm{T}}$ is so far only a symbol used to define the Breit frame transverse component, and it has yet to be related to physical quantities. Such parameterization of the final hadron momentum is convenient for some purposes such as in factorization derivations. Vector $\mathbf{q}_{\mathrm{T}}$ in the Breit frame is

$$
\mathbf{q}_{\mathrm{T}} \equiv-\frac{\mathbf{P}_{\mathrm{B}, \mathrm{b}, \mathrm{T}}}{z_{\mathrm{N}}}=-\frac{q_{\mathrm{b}}^{-} \mathbf{P}_{\mathrm{B}, \mathrm{b}, \mathrm{T}}}{P_{\mathrm{B}, \mathrm{b}}^{-}} .
$$

Note the minus sign. The momentum fraction $z_{\mathrm{N}}$ is related to the kinematical parameter $z_{\mathrm{h}}$ by

$$
z_{\mathrm{N}}=\frac{Q^{4} x_{\mathrm{N}} z_{\mathrm{h}}\left(1 \pm \sqrt{1-\frac{4 M^{2} M_{\mathrm{B}}^{2} x_{\mathrm{Bj}}^{2}\left(Q^{4}+x_{\mathrm{N}}^{2} M^{2} \mathbf{q}_{\mathrm{T}}^{2}\right)}{Q^{8} z_{\mathrm{h}}^{2}}}\right)}{2 x_{\mathrm{Bj}}\left(Q^{4}+x_{\mathrm{N}}^{2} M^{2} \mathbf{q}_{\mathrm{T}}^{2}\right)} \stackrel{\text { Fixed } \mathrm{x}_{\mathrm{N}}, \mathrm{z}_{\mathrm{h}}, \mathrm{q}_{\mathrm{T}}}{=} z_{\mathrm{h}}\left(1+O\left(\frac{m^{4}}{Q^{4}}\right)\right)
$$

Note that the relationship between $z_{\mathrm{N}}$ and $z_{\mathrm{h}}$ is generally double valued. The expansion after the far right equals sign in eq. (2.22) is for the "+" solution, which in conventional treatment of SIDIS corresponds to the current fragmentation region.

Another way to establish the connection of the momentum fractions $z_{\mathrm{h}}$ and $z_{\mathrm{N}}$ is by utilizing the transverse mass $M_{\mathrm{B}, \mathrm{T}}$ and experimentally measured $\mathbf{P}_{\mathrm{B}, \mathrm{b}, \mathrm{T}}$. Let us start by using the definition of $z_{\mathrm{h}}$ from eq. (2.3):

$$
z_{\mathrm{h}}=\frac{x_{\mathrm{Bj}} z_{\mathrm{N}}}{x_{\mathrm{N}}}\left(1+\frac{x_{\mathrm{N}}^{2} M^{2} M_{\mathrm{B}, \mathrm{T}}^{2}}{z_{\mathrm{N}}^{2} Q^{4}}\right) .
$$

The inverse, for the "+" solution, is

$$
z_{\mathrm{N}}=\frac{x_{\mathrm{N}} z_{\mathrm{h}}}{2 x_{\mathrm{Bj}}}\left(1+\sqrt{1-\frac{4 M^{2} M_{\mathrm{B}, \mathrm{T}}^{2} x_{\mathrm{Bj}}^{2}}{Q^{4} z_{\mathrm{h}}^{2}}}\right) \approx z_{\mathrm{h}}
$$

Note that eq. (2.22) and eq. (2.24) are related upon substitution from eq. (2.21). See also ref. [25, eq. (2.12)]. Note that $x_{\mathrm{N}}$ is a function of $x_{\mathrm{Bj}}, Q$, and $M$, but we will sometimes 
keep it in formulas in order to minimize the size of expressions such as in eq. (2.24) rather than writing everything explicitly in terms of $x_{\mathrm{Bj}}, Q$, and $M$.

Now we use the Lorentz transformations of light-cone vectors between the photon (e.g. Breit) and hadron frames: for this we have presented a detailed treatment how to transform a four vector $V$ from the Breit frame to the hadron frame in a light cone representation in appendix B (see eqs. (B.2)-(B.4)). We use these results in the limit that the masses are small relative to $Q$ which are

$$
\begin{aligned}
V_{\mathrm{H}}^{+} & \approx V_{\mathrm{b}}^{+}+\frac{\mathbf{q}_{\mathrm{T}}^{2}}{Q^{2}} V_{\mathrm{b}}^{-}+\frac{\sqrt{2}}{Q} \mathbf{q}_{\mathrm{T}} \cdot \mathbf{V}_{\mathrm{b}, \mathrm{T}}, \\
V_{\mathrm{H}}^{-} & \approx V_{\mathrm{b}}^{-}, \\
\mathbf{V}_{\mathrm{H}, \mathrm{T}} & \approx \mathbf{V}_{\mathrm{b}, \mathrm{T}}+\mathbf{q}_{\mathrm{T}} \frac{\sqrt{2} V_{\mathrm{b}}^{-}}{Q} .
\end{aligned}
$$

Now using eq. (2.17) in eq. (2.27), yields

$$
\mathbf{q}_{\mathrm{H}, \mathrm{T}} \approx \mathbf{q}_{\mathrm{T}} .
$$

Finally using eq. (2.21) and eq. (2.22) we conclude that

$$
\mathbf{q}_{\mathrm{H}, \mathrm{T}} \approx-\frac{\mathbf{P}_{\mathrm{B}, \mathrm{b}, \mathrm{T}}}{z_{\mathrm{h}}} \approx \mathbf{q}_{\mathrm{T}},
$$

which means that up to $m / Q$-suppressed corrections the transverse vector $\mathbf{q}_{\mathrm{T}}$ introduced in eq. (2.20) to parametrize the final hadron transverse momentum in the Breit frame is equal to the hadron frame photon transverse momentum $\mathbf{q}_{\mathrm{H}, \mathrm{T}}$, which in turn is equal to $-\mathbf{P}_{\mathrm{B}, \mathrm{b}, \mathrm{T}} / z_{\mathrm{h}}$. Here as usual, the $\approx$ symbol means neglecting $m / Q$-suppressed corrections.

\subsection{The SIDIS cross section}

In this sub-section we consider the cross-section of the SIDIS process, and translate past results into an updated language, motivated by current research efforts; e.g. to interpret moderate-to-low $Q$ cross sections. In particular we establish an extension for the SIDIS cross section to where the structure functions depend not only on $\mathbf{P}_{\mathrm{B}, \mathrm{T}}, x_{N}$ and $z_{N}$ but also on hadron masses $M$ and $M_{B}$. In order to show/demonstrate this, we briefly review the definitions of both the DIS and SIDIS cross sections.

We start by writing down the total DIS cross section,

$$
E^{\prime} \frac{\mathrm{d} \sigma^{\text {tot }}}{\mathrm{d}^{3} \mathbf{l}^{\prime}}=\frac{2 \alpha_{\mathrm{em}}^{2}}{\left(s-M^{2}\right) Q^{4}} L_{\mu \nu} W_{\mathrm{tot}}^{\mu \nu}
$$

This fixes the normalization convention for the hadron and lepton tensor combination $L_{\mu \nu} W_{\text {tot }}^{\mu \nu}$, where the "tot"-subindex indicates that this is conventional DIS: totally inclusive in all final state hadrons. Recall figure 1 and eq. (2.1) for our momentum labeling. The leptonic tensor is defined in the usual way:

$$
L_{\mu \nu}=2\left(l_{\mu} l_{\nu}^{\prime}+l_{\mu}^{\prime} l_{\nu}-g_{\mu \nu} l \cdot l^{\prime}\right)
$$


and the totally inclusive hadronic tensor is

$$
W_{\mathrm{tot}}^{\mu \nu}(P, q) \equiv 4 \pi^{3} \sum_{X} \delta^{(4)}\left(P+q-P_{X}\right)\left\langle P, S\left|j^{\mu}(0)\right| X\right\rangle\left\langle X\left|j^{\nu}(0)\right| P, S\right\rangle .
$$

Here, the $\sum_{X}$ symbol is a sum over all possible final states $|X\rangle$, including invariant integrals,

$$
\int \frac{\mathrm{d}^{3} \boldsymbol{p}_{i}}{2 E_{p_{i}}(2 \pi)^{3}} \cdots
$$

over the momentum of each final state particle $p_{i}$. The incoming hadron has a polarization specified by $S$.

Now we write down the SIDIS cross section, differential in the momentum of the final state hadron of type $B$, which can be expressed in terms of the leptonic and hadronic tensors as follows:

$$
4 P_{\mathrm{B}}{ }^{0} E^{\prime} \frac{\mathrm{d} \sigma}{\mathrm{d}^{3} \mathbf{l}^{\prime} \mathrm{d}^{3} \mathbf{P}_{\mathrm{B}}}=\frac{2 \alpha_{\mathrm{em}}^{2}}{\left(s-M^{2}\right) Q^{4}} L_{\mu \nu} W_{\mathrm{SIDIS}}^{\mu \nu} .
$$

Similarly as to the DIS case, this fixes our normalization conventions for SIDIS, and gives a SIDIS hadronic tensor:

$$
W_{\text {SIDIS }}^{\mu \nu}\left(P, q, P_{\mathrm{B}}\right) \equiv \sum_{X} \delta^{(4)}\left(P+q-P_{\mathrm{B}}-P_{X}\right)\left\langle P, S\left|j^{\mu}(0)\right| P_{\mathrm{B}}, X\right\rangle\left\langle P_{\mathrm{B}}, X\left|j^{\nu}(0)\right| P, S\right\rangle .
$$

The same meaning applies to $\sum_{X}$ as in the totally inclusive case. $\left|P_{\mathrm{B}}, X\right\rangle$ is a final state with at least one identified hadron of type $B$. The sum over $X$ includes a sum over any number of other final state particles, including other type- $B$ hadrons. Each separate type$B$ hadron in an event is counted, in accordance with the definition of an inclusive cross section. More details on the SIDIS differential cross sections are given in appendix $\mathrm{C}$.

The hadronic tensors $W_{\text {tot }}^{\mu \nu}(P, q)$ and $W_{\text {SIDIS }}^{\mu \nu}\left(P, q, P_{\mathrm{B}}\right)$ are the most convenient objects to work with theoretically because they are directly related to hadronic matrix elements of the electromagnetic current operator, and they are defined without reference to conventions associated with choices of reference frames etc, so we will organize our structure function analysis around them.

Now we establish the relationship between the semi-inclusive and totally inclusive cross sections (see [26, Chapt. VII]):

$$
\sum_{B} \int \mathrm{d}^{3} \mathbf{P}_{B} \frac{\mathrm{d} \sigma}{\mathrm{d}^{3} \mathbf{P}_{B}}=\langle N\rangle \sigma^{\mathrm{tot}},
$$

where $\langle N\rangle$ is the total average particle multiplicity, and the sum is over all particle types. Thus,

$$
\sum_{B} \int \frac{\mathrm{d}^{2} \mathbf{P}_{\mathrm{B}, \mathrm{b}, \mathrm{T}} \mathrm{d} P_{\mathrm{B}}^{z}}{4 P_{\mathrm{B}}^{0}} W_{\text {SIDIS }}^{\mu \nu}=\sum_{B} \int \frac{\mathrm{d}^{2} \mathbf{P}_{\mathrm{B}, \mathrm{b}, \mathrm{T}} \mathrm{d} z_{\mathrm{N}}}{4 z_{\mathrm{N}}} W_{\text {SIDIS }}^{\mu \nu}=\langle N\rangle W_{\mathrm{tot}}^{\mu \nu} .
$$

Note that the integration measure in eq. (2.36) is Lorentz invariant, although we will continue to specify a photon frame for the components, both for definiteness and because $z_{\mathrm{N}}$ is defined in terms of a photon frame momentum fraction. 
Using the standard definitions of the structure function decompositions for $W_{\text {tot }}^{\mu \nu}$ and $W_{\text {SIDIS }}^{\mu \nu}$, we express the SIDIS cross section the in the Breit frame (or any photon frame),

$$
\frac{\mathrm{d} \sigma}{\mathrm{d} x_{\mathrm{Bj}} \mathrm{d} y \mathrm{~d} \psi \mathrm{d} z_{\mathrm{h}} \mathrm{d}^{2} \mathbf{P}_{\mathrm{B}, b, \mathrm{~T}}}=\frac{\alpha_{\mathrm{em}}^{2} y}{4 z_{\mathrm{h}} x_{\mathrm{Bj}} Q^{2}(1-\varepsilon)} \frac{1}{\sqrt{1-\frac{4 M^{2} x_{\mathrm{Bj}}^{2} M_{\mathrm{B}, \mathrm{T}}^{2}}{Q^{4} z_{\mathrm{h}}^{2}}}}\left[F_{T}+\varepsilon F_{L}+\text { Pol. Dep. }\right],
$$

where $F_{T}$ and $F_{L}$ are definitions generalized from the inclusive case to SIDIS and "Pol. Dep." indicates the presence of polarization and azimuthal dependent terms ( see appendix $\mathrm{C}$ for details). The Jacobian factor in eq. (2.37), that contains the final hadron transverse mass, can be expressed in terms of combinations of $z_{\mathrm{N}}$ and $z_{\mathrm{h}}$, but we keep the square root factors explicit to highlight the dependence on transverse momentum via $M_{\mathrm{B}, \mathrm{T}}^{2}$ at fixed $z_{\mathrm{h}}$.

Now, to reproduce the equations of ref. [20], one may define barred structure functions:

$$
\bar{F}_{j}=\frac{1}{4 z_{\mathrm{h}}\left(1+\frac{\gamma^{2}}{2 x_{\mathrm{Bj}}}\right)} \frac{F_{j}}{\sqrt{1-\frac{4 M^{2} x_{\mathrm{Bj}}^{2} M_{\mathrm{B}, \mathrm{T}}^{2}}{Q^{4} z_{\mathrm{h}}^{2}}}},
$$

with $\gamma \equiv 2 M x_{\mathrm{Bj}} / Q$. Substituting eq. (2.37) into eq. (2.37) gives

$$
\frac{\mathrm{d} \sigma}{\mathrm{d} x_{\mathrm{Bj}} \mathrm{d} y \mathrm{~d} \psi \mathrm{d} z_{\mathrm{h}} \mathrm{d}^{2} \mathbf{P}_{\mathrm{B}, \mathrm{b}, \mathrm{T}}}=\frac{\alpha_{\mathrm{em}}^{2} y}{x_{\mathrm{Bj}} Q^{2}(1-\varepsilon)}\left(1+\frac{\gamma^{2}}{2 x_{\mathrm{Bj}}}\right)\left[\bar{F}_{T}+\varepsilon \bar{F}_{L}+\text { Pol. Dep. }\right] \text {, }
$$

where now $[20$, eq. (2.7)] can be used to fill in the remaining polarization and $\phi$-dependent structure functions.

We emphasize that eq. (2.37) is an exact expression, where no approximation has been made. Thus, the structure functions depend not only on $\mathbf{P}_{\mathrm{B}, \mathrm{T}}, x_{N}$ and $z_{N}$ but also on hadron masses $M$ and $M_{B}$. It is important to highlight the role of the final hadron mass, which couples transverse momentum to other kinematical variables, as can be seen from the Jacobian in eq. (2.37). The barred normalization convention in eq. (2.38) is defined so that structure functions exactly obey a particularly convenient energy sum rule found in ref. [20, eqs. (2.18)-(2.21)]:

$$
\sum_{B} \int \mathrm{d} z_{\mathrm{h}} \mathrm{d}^{2} \mathbf{P}_{\mathrm{B}, \mathrm{b}, \mathrm{T}} z_{\mathrm{h}} \bar{F}_{j}=F_{j}^{\mathrm{tot}}
$$

Note that the general derivation of the sum rule eq. (2.40) does not follow trivially from that presented in ref. [20], due to presence of the factor containing $M_{\mathrm{B}, \mathrm{T}}^{2}$ in eq. (2.38). A new derivation of eq. (2.40) is presented in appendix D.

\section{Kinematical approximations}

Since we have not discussed the theory underlying the structure functions, all small mass approximations mentioned so far are unambiguously kinematical. For example, the usual 
$x_{\mathrm{N}} \approx x_{\mathrm{Bj}}$ and $z_{\mathrm{N}} \approx z_{\mathrm{h}}$ follow from expanding in $x_{\mathrm{Bj}}^{2} M^{2} / Q^{2}$ :

$$
\begin{aligned}
x_{\mathrm{N}}= & x_{\mathrm{Bj}}\left[1-\frac{x_{\mathrm{Bj}}^{2} M^{2}}{Q^{2}}+O\left(\frac{x_{\mathrm{Bj}}^{4} M^{4}}{Q^{4}}\right)\right] \\
z_{\mathrm{N}}= & z_{\mathrm{h}}\left[1-\frac{x_{\mathrm{Bj}}^{2} M^{2}}{Q^{2}}\left(1+\frac{\mathbf{P}_{\mathrm{B}, \mathrm{b}, \mathrm{T}}^{2}}{z_{\mathrm{h}}^{2} Q^{2}}\right)+\left(\frac{x_{\mathrm{Bj}}^{2} M^{2}}{Q^{2}}\right)^{2}\left(\frac{\mathbf{P}_{\mathrm{B}, \mathrm{b}, \mathrm{T}}^{2}}{z_{\mathrm{h}}^{2} Q^{2}}-\frac{\mathbf{P}_{\mathrm{B}, \mathrm{b}, \mathrm{T}}^{4}}{z_{\mathrm{h}}^{4} Q^{4}}+2-\frac{M_{\mathrm{B}}^{2}}{z_{\mathrm{h}}^{2} M^{2} x_{\mathrm{Bj}}^{2}}\right)\right. \\
& \left.+O\left(\frac{x_{\mathrm{Bj}}^{6} M^{6}}{Q^{6}}\right)\right]
\end{aligned}
$$

If hadron masses are neglected, then $P$ and $P_{\mathrm{B}}$ become the approximate $\tilde{P}$ and $\tilde{P_{\mathrm{B}}}$, which we define as

$$
\begin{aligned}
\tilde{P}_{\mathrm{b}} & =\left(\frac{Q}{x_{\mathrm{Bj}} \sqrt{2}}, 0, \mathbf{0}_{\mathrm{T}}\right), \\
\tilde{P}_{\mathrm{B}, \mathrm{b}} & =z_{\mathrm{h}}\left(\frac{\mathbf{q}_{\mathrm{T}}^{2}}{\sqrt{2} Q}, \frac{Q}{\sqrt{2}},-\mathbf{q}_{\mathrm{T}}\right),
\end{aligned}
$$

that is, eq. (2.18) and eq. (2.19) but with all hadron masses set equal to zero. In the most common treatments of SIDIS, $P$ and $P_{\mathrm{B}}$ in eqs. (C.2)-(C.3) are replaced with $\tilde{P}$ and $\tilde{P}_{\mathrm{B}}$, and $x_{\mathrm{N}}$ and $z_{\mathrm{N}}$ are replaced with $x_{\mathrm{Bj}}$ and $z_{\mathrm{h}}$ inside the structure functions, which is a good approximation in the $m / Q \rightarrow 0$ limit as long as the structure functions are reasonably smooth functions of $x_{\mathrm{N}}$ and $z_{\mathrm{N}}$. In ref. [23] that was called the massless target approximation (MTA) for inclusive DIS, and we extend this to SIDIS. In this case, the hadronic tensors from eqs. (C.2)-(C.3) become,

$$
\begin{aligned}
\tilde{W}_{\mathrm{tot}}^{\mu \nu}= & \left(-g^{\mu \nu}+\frac{q^{\mu} q^{\nu}}{q^{2}}\right) \mathcal{F}_{1}^{\mathrm{tot}}\left(x_{\mathrm{Bj}}, Q^{2}\right)+\frac{\left(\tilde{P}^{\mu}-q^{\mu} \frac{\tilde{P} \cdot q}{q^{2}}\right)\left(\tilde{P}^{\nu}-q^{\nu} \frac{\tilde{P} \cdot q}{q^{2}}\right)}{\tilde{P} \cdot q} \mathcal{F}_{2}^{\mathrm{tot}}\left(x_{\mathrm{Bj}}, Q^{2}\right) \\
& + \text { Pol. Dep. } \\
\tilde{W}_{\mathrm{SIDIS}}^{\mu \nu}= & \left(-g^{\mu \nu}+\frac{q^{\mu} q^{\nu}}{q^{2}}\right) \mathcal{F}_{1}\left(x_{\mathrm{Bj}}, Q^{2}, z_{\mathrm{h}}, \tilde{\mathbf{P}}_{\mathrm{B}, \mathrm{b}, \mathrm{T}}\right) \\
& +\frac{\left(\tilde{P}^{\mu}-q^{\mu} \frac{\tilde{P} \cdot q}{q^{2}}\right)\left(\tilde{P}^{\nu}-q^{\nu} \frac{\tilde{P} \cdot q}{q^{2}}\right)}{\tilde{P} \cdot q} \mathcal{F}_{2}\left(x_{\mathrm{Bj}}, Q^{2}, z_{\mathrm{h}}, \tilde{\mathbf{P}}_{\mathrm{B}, \mathrm{b}, \mathrm{T}}\right)+\text { Pol. Dep. }
\end{aligned}
$$

Extracting the structure functions in eqs. (3.5)-(3.6) requires, instead of eqs. (C.11)(C.12), the following projectors

$$
\tilde{\mathrm{P}}_{1}^{\mu \nu}=-\frac{1}{2} \mathrm{P}_{g}^{\mu \nu}+\frac{2 x_{\mathrm{Bj}}^{2}}{Q^{2}} \tilde{\mathrm{P}}_{P P}^{\mu \nu}, \quad \tilde{\mathrm{P}}_{2}^{\mu \nu}=\frac{12 x_{\mathrm{Bj}}^{3}}{Q^{2}} \tilde{\mathrm{P}}_{P P}^{\mu \nu}-x_{\mathrm{Bj}} \mathrm{P}_{g}^{\mu \nu},
$$

where $\tilde{\mathrm{P}}_{P P}^{\mu \nu}=\tilde{P}^{\mu} \tilde{P}^{\nu}$. Our eq. (3.5) coincides with ref. [23, eq. (18)], with the calligraphic notation explained there. Equation (3.6) is the analogous approximation for the SIDIS 

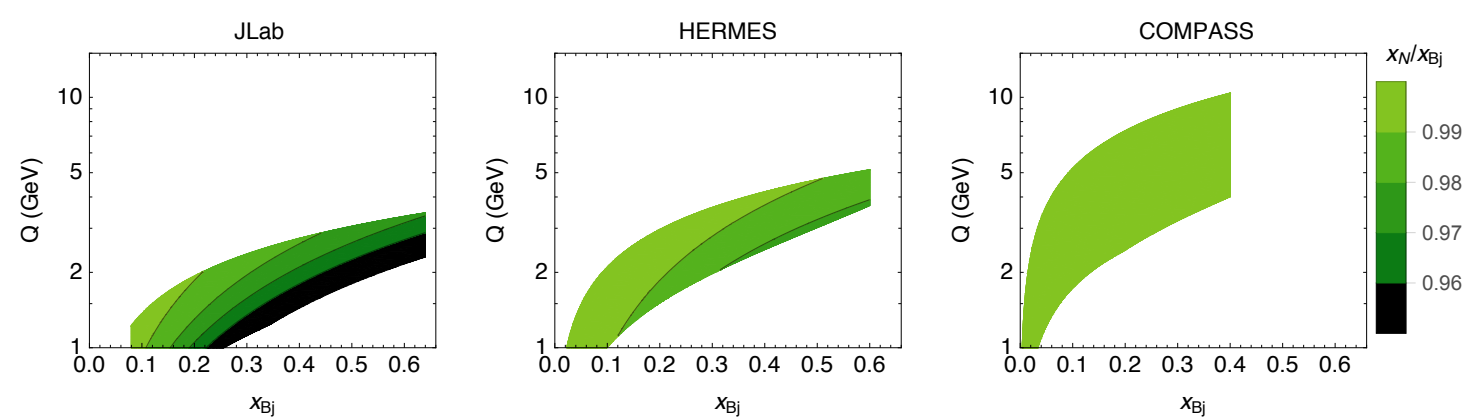

Figure 3. The kinematic regions of $Q$ and $x_{\mathrm{Bj}}$ covered by JLab 12 (left panel), HERMES (central panel) and COMPASS (right panel). The shaded areas are obtained by applying the appropriate experimental cuts in each case, as reported in refs. [6, 27, 28]. These plots show that $Q$ and $x_{\mathrm{Bj}}$ are strongly correlated: large values of $x_{\mathrm{Bj}}$ can only be accessed when $Q$ is sufficiently large; conversely, when $Q$ is relatively small, only limited values of $x_{\mathrm{Bj}}$ can be reached. The values of $x_{N} / x_{\mathrm{Bj}}$, as obtained using eq. (2.2), are color-coded: the lightest shade corresponds to values very close to one, while darker shades correspond to regions where the ratio $x_{N} / x_{\mathrm{Bj}}$ increasingly deviates from 1 and the quality of the MHA deteriorates. Notice that, while mass corrections are more important for JLab 12 kinematics, for all three experiments we consider here, the value $x_{\mathrm{N}} / x_{\mathrm{Bj}}$ remains very close to 1 to a very good approximation.

cross section. The advantage of usage of MHA is that it greatly simplifies kinematical relations at large $Q$. The ratios

$$
\frac{x_{\mathrm{N}}}{x_{\mathrm{Bj}}}, \quad \frac{z_{\mathrm{N}}}{z_{\mathrm{h}}}
$$

are measures of the quality of the MHA approximation. They must not deviate too much from 1 if the standard massless approximations are to be considered valid. ${ }^{6}$

This discussion exhausts the approximations that can be assessed entirely independently of questions about the partonic dynamics responsible for the behavior of the structure functions themselves. Thus, to test the quality of the MHA-which deteriorates as the ratios in eq. (3.8) deviate from 1-we study these quantities for some realistic experimental scenarios.

In figure 3 we display the $\left(Q, x_{\mathrm{Bj}}\right)$ kinematic coverage of three fixed target SIDIS experiments: JLab 12 (11 GeV electron beam), HERMES (27.5 GeV electron beam) and COMPASS ( $160 \mathrm{GeV}$ muon beam). The shaded regions are obtained by applying the appropriate experimental cuts in each case, as reported in refs. [6, 27, 28]. Notice that the JLab 12 kinematics covers a very wide range of $x_{\mathrm{Bj}}$ values, well above 0.6 , but it is limited to intermediate/small values of $Q$. Instead, the COMPASS kinematics reaches up to much larger values of $Q$, but the accessible range of $x_{\mathrm{Bj}}$ is confined to values no larger than 0.4. In each plot, the values of the ratio $x_{\mathrm{N}} / x_{\mathrm{Bj}}$, eq. (2.2), are color coded: darker shades represent regions where $x_{\mathrm{N}} / x_{\mathrm{Bj}}$ deviates from 1 and thus the MHA approximation deteriorates. As expected mass corrections are more important at large values of $x_{\mathrm{Bj}}$ and small values of $Q$. All three experiments we consider here, have $x_{\mathrm{N}} / x_{\mathrm{Bj}} \approx 1$ within $5 \%$, and thus massless hadron approximation remain a good approximation for all three experiments provided that experimental errors are not infinitesimal.

\footnotetext{
${ }^{6}$ See section 5 for some examples.
} 

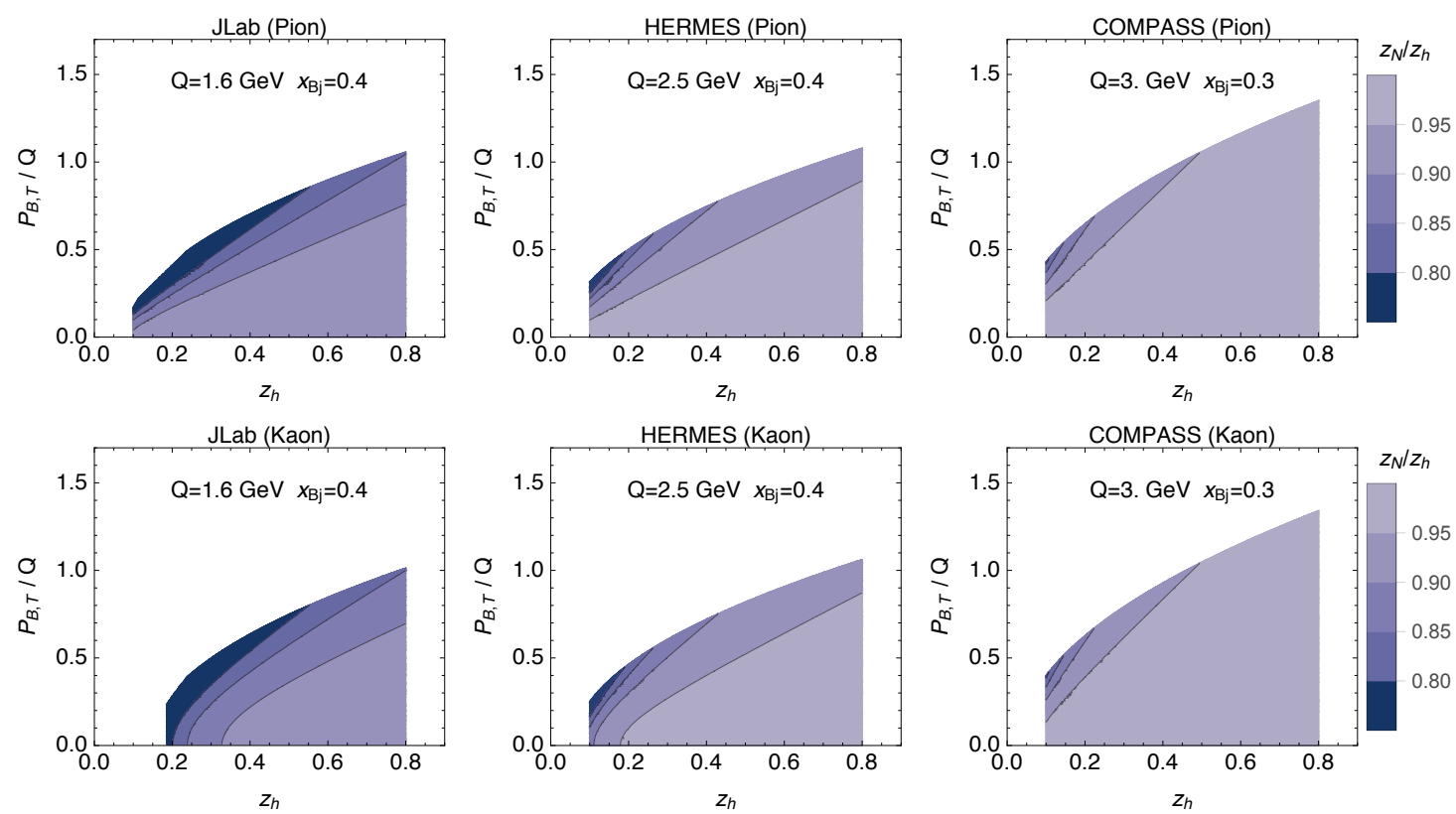

Figure 4. The ratio $z_{N} / z_{h}$, eq. (2.24), is represented over the kinematic coverage in $\left(z_{h}, P_{B, T} / Q\right)$ for JLab 12 (left panels), HERMES (central panels) and COMPASS (right panels), at some fixed values of $x_{\mathrm{Bj}}$ and $Q$, as indicated in the plot title. Appropriate experimental cuts, as reported in refs. [6, 27, 28], are applied in each case. The values of $z_{N} / z_{h}$, for pion production (upper panels) and kaon production (lower panels) are obtained using eq. (2.24) and are color-coded: the lightest shade corresponds to values very close to one, while darker shades correspond to regions where the ratio $z_{N} / z_{h}$ increasingly deviates from 1 and the quality of the MHA deteriorates. Notice how deviations from 1 are more sizable as compared to those of $x_{\mathrm{N}} / x_{\mathrm{Bj}}$ in figure 3 , particularly in the JLab case.

Figure 4 shows the ratio $z_{\mathrm{N}} / z_{\mathrm{h}}$, within the $\left(z_{\mathrm{h}}, P_{B, T} / Q\right)$ kinematic coverage of the three experiments. Again darker shades represent larger deviations from 1 which, in this case, are more significant than for $x_{\mathrm{Bj}} / x_{\mathrm{N}}$, especially at JLab kinematics where considerably wide regions of phase space have $z_{N} / z_{h}<0.9$. This demonstrates the significance of mass correction effects in eq. (3.2). Refs. [25, 29] made first attempts to incorporate kinematical improvements to collinear QCD factorization by keeping $M_{\mathrm{B}}^{2}$ in kinematical factors. They point out the importance of this for moderate-to-low $Q$ SIDIS. However, they explicitly drop $\mathbf{P}_{\mathrm{B}, \mathrm{b}, \mathrm{T}}^{2}$-dependence in an attempt to stay within a collinear factorization framework. Note from eq. (3.2), however, that it is not consistent with collinear factorization power counting to simultaneously retain $M^{2}$ and $M_{\mathrm{B}}^{2}$ dependent kinematical power corrections while neglecting $\mathbf{P}_{\mathrm{B}, \mathrm{b}, \mathrm{T}}^{2}$ dependent corrections, even for $\mathbf{P}_{\mathrm{B}, \mathrm{b}, \mathrm{T}}^{2} \sim m^{2}$. The first nonvanishing $M_{\mathrm{B}}$-dependent correction term

$$
\frac{M_{\mathrm{B}}^{2}}{z_{\mathrm{h}}^{2} M^{2} x_{\mathrm{Bj}}^{2}}\left(\frac{x_{\mathrm{Bj}}^{2} M^{2}}{Q^{2}}\right)^{2}
$$

is the same size as the

$$
\frac{\mathbf{P}_{\mathrm{B}, \mathrm{b}, \mathrm{T}}^{2}}{z_{\mathrm{h}}^{2} Q^{2}}\left(\frac{x_{\mathrm{Bj}}^{2} M^{2}}{Q^{2}}\right)
$$


term when $\mathbf{P}_{\mathrm{B}, \mathrm{b}, \mathrm{T}}^{2}$ is small. And, eq. (3.10) is actually the dominant power-correction term when $\mathbf{P}_{\mathrm{B}, \mathrm{b}, \mathrm{T}}^{2} / z_{\mathrm{h}}^{2}$ approaches order $Q$. The difficulty is that collinear factorization methods only characterize dependence on light-cone momentum fractions of the final state hadron, like $z_{\mathrm{N}}$, with only $z_{\mathrm{h}}, Q$, and $x_{\mathrm{Bj}}$ known. This is not a problem if keeping only the first term in the expansion on the right of eq. (3.2) is valid. But the exact $z_{\mathrm{N}}$ requires knowledge not just of $M_{\mathrm{B}}$ and $M$, but also of (both small and large) $\mathbf{P}_{\mathrm{B}, \mathrm{b}, \mathrm{T}}^{2} / z_{\mathrm{h}}^{2}$. So if it turns out that final state mass effects are large enough that they have to be accounted for, then it must be done in combination with an account of small transverse momentum dependence effects (e.g., TMD factorization), not independently of it.

\section{Mapping SIDIS kinematics and partonic subprocesses}

In this section we will present the main results of our work. We will establish the correspondence between specific underlying partonic pictures of the SIDIS process and kinematical regions of experimentally observed cross section.

So far, we have only discussed definitions and relativistic kinematics, with no mention at all of partons or dynamics. The question now is the following: assuming that the configuration of initial and final hadrons is the result of scattering and fragmentation by small-mass constituents (i.e., partons), what are the possible kinematical configurations of those constituents, given a set of assumptions about their intrinsic properties? For now, we do not necessarily identify these partons with a particular theoretical approach or even real QCD, though ultimately we have that in mind.

This kind of very general partonic picture is illustrated in figure 5. We start by exploring the possibility that the produced hadron is collinear to an outgoing parton (a "current" hadron). We need clear steps for asking how reasonable it is to assume that a given external kinematical configuration for measured hadrons maps to current region partonic kinematics. The incoming hadron and its remnants are represented by the lower blob while the final state hadron emerges from a final state blob at the top of the diagram. Dashed lines represent the flow of momentum. It is very important for the discussion below to understand that they do not necessarily represent single quarks or gluons, and in reality they may correspond to groups of particles. What is important for us is only the flow of fourmomentum through the process. Moreover, it is assumed that the momenta of these lines are known exactly and are never approximated. Thus, the graphs should not be viewed as Feynman graphs used in the calculation of amplitudes, but rather as charts of momentum flow. Although the word "parton" often implies a massless on-shell approximation for single particle lines, to keep language reasonably simple, we will nevertheless continue to call these dashed lines "partons." The picture in figure 5 does imply that quantities like $\left|k_{\mathrm{i}}^{2}\right|$ and $\left|k_{\mathrm{f}}^{2}\right|$ are small, and much of the discussion in this section will be about addressing the question of what is meant by "small." So to summarize, "partonic" dashed lines represent the flow of momentum with small invariant energy. In practical situations, they will often turn out to refer to actual quark and/or gluon lines, but they do not need to generally.

The partonic subprocess in figure 5 is marked off in a blue box. A black dot indicates the parton we associate with an observed hadron. The momentum $k_{\mathrm{i}}$ is the incoming 


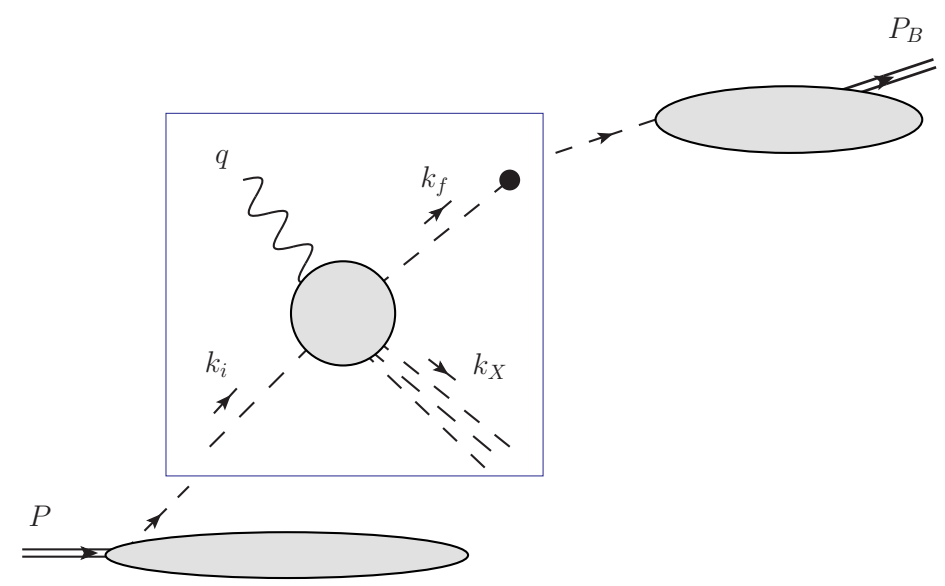

Figure 5. Momentum labeling in the partonic subprocess. The lower blob represent the incoming hadron. The diagram in the square is the partonic subprocess of interaction of partons and the virtual photon. Dashed lines represent the flow of momentum. The process of fragmentation of the outgoing parton into the observed hadron B is represented by the upper blob.

struck parton momentum, and there is at least one hadronizing parton $k_{\mathrm{f}}$. The $k_{\mathrm{X}}$ momentum labels the total momentum of all other unobserved partons combined. Outside the box in figure 5 , the position of the hadron implies a current region picture, though an analogous picture of course applies to the target region case. We ask questions about partonic regions in the context of the steps needed to factorize graphical structure in a manner consistent with particular partonic pictures. Our general view of factorization is based on that of Collins $[11,30]$ and collaborators, though the same statements apply to most other approaches.

We are interested in the kinematics of the $k_{\mathrm{i}}+q \rightarrow k_{\mathrm{f}}+k_{X}$ subprocess and how closely it matches the overall $P+q \rightarrow P_{\mathrm{B}}+X$ process under very general assumptions. Specific realizations of the partonic subprocess, each of which can contribute to a different kinematical region, are shown in figure 6 . We will analyze the subprocess in the Breit frame and write

$$
k_{\mathrm{i}}^{\mathrm{b}}=\left(\frac{Q}{\hat{x}_{\mathrm{N}} \sqrt{2}}, \frac{\hat{x}_{\mathrm{N}}\left(k_{\mathrm{i}}^{2}+\mathbf{k}_{\mathrm{i}, \mathrm{b}, \mathrm{T}}^{2}\right)}{\sqrt{2} Q}, \mathbf{k}_{\mathrm{i}, \mathrm{b}, \mathrm{T}}\right), \quad k_{\mathrm{f}}^{\mathrm{b}}=\left(\frac{\mathbf{k}_{\mathrm{f}, \mathrm{b}, \mathrm{T}}^{2}+k_{\mathrm{f}}^{2}}{\sqrt{2} \hat{z}_{\mathrm{N}} Q}, \frac{\hat{z}_{\mathrm{N}} Q}{\sqrt{2}}, \mathbf{k}_{\mathrm{f}, \mathrm{b}, \mathrm{T}}\right) .
$$

Hats always indicate a partonic kinematical variable, whereas $\xi$ and $\zeta$ are momentum fractions. We have defined the Breit frame momentum fractions and Breit frame $\hat{x}_{N}, \hat{z}_{N}$ analogous to $x_{\mathrm{N}}$ and $x_{\mathrm{Bj}}$ :

$$
k_{\mathrm{i}}^{+} \equiv \xi P_{\mathrm{b}}^{+}, \quad P_{\mathrm{B}, \mathrm{b}}^{-} \equiv \zeta k_{\mathrm{f}}^{-}, \quad \hat{x}_{\mathrm{N}} \equiv-\frac{q_{\mathrm{b}}^{+}}{k_{\mathrm{i}, \mathrm{b}}^{+}}=\frac{x_{\mathrm{N}}}{\xi}, \quad \hat{z}_{\mathrm{N}} \equiv \frac{k_{\mathrm{f}, \mathrm{b}}^{-}}{q_{\mathrm{b}}^{-}}=\frac{z_{\mathrm{N}}}{\zeta} .
$$

We will write the transverse momentum as

$$
\mathbf{k}_{\mathrm{f}, \mathrm{b}, \mathrm{T}}=-\hat{z}_{\mathrm{N}} \mathbf{q}_{\mathrm{T}}+\delta \mathbf{k}_{\mathrm{T}}
$$


In the hadron frame, eq. (B.2) gives

$$
\mathbf{k}_{\mathrm{f}, \mathrm{H}, \mathrm{T}}=\delta \mathbf{k}_{\mathrm{T}}+\text { Power Suppressed }
$$

so $\delta \mathbf{k}_{\mathrm{T}}$ is good for characterizing an intrinsic relative transverse momentum in the large $Q$ limit; in eq. (4.1) intrinsic transverse momentum is $\delta \mathbf{k}_{\mathrm{T}}$ when $q_{\mathrm{T}}=0$. For nearly on-shell partons,

$$
\left|k_{\mathrm{i}}^{2}\right|,\left|k_{\mathrm{f}}^{2}\right|=O\left(m^{2}\right) .
$$

In the limit where $m \ll Q$ and $x_{\mathrm{Bj}}, z_{\mathrm{h}}, q_{\mathrm{T}}$ are fixed, the outgoing parton is exactly aligned with the observed hadron so long as

$$
\delta k_{\mathrm{T}}^{2}=O\left(m^{2}\right) .
$$

For fixed $\hat{x}_{\mathrm{N}}, \hat{z}_{\mathrm{N}}$ and $q_{\mathrm{T}}^{2}, k_{\mathrm{X}}^{2}$ is calculable from momentum conservation,

$$
k_{\mathrm{X}}^{2}=\left(k_{\mathrm{i}}+q-k_{\mathrm{f}}\right)^{2} .
$$

It will also be useful to define a momentum variable

$$
k \equiv k_{\mathrm{f}}-q .
$$

It is sometimes useful to have $k$ in terms of $k_{X}^{2}$ instead of $\hat{z}_{\mathrm{N}}$. For example, in the special case that $k_{\mathrm{i}}^{2}=k_{\mathrm{f}}^{2}=\mathbf{k}_{\mathrm{i}, \mathrm{b}, \mathrm{T}}^{2}=\delta \mathbf{k}_{\mathrm{T}}^{2}=0$

$$
\begin{aligned}
k_{\mathrm{b}}^{+} & =\frac{Q}{\sqrt{2}}\left(1+\frac{q_{\mathrm{T}}^{2}}{Q^{2}}\left(\frac{1-\hat{x}_{\mathrm{N}}\left(1+k_{\mathrm{X}}^{2} / Q^{2}\right)}{1-\hat{x}_{\mathrm{N}}\left(1-q_{\mathrm{T}}^{2} / Q^{2}\right)}\right)\right)=\frac{Q}{\sqrt{2}}\left(1+\frac{q_{\mathrm{T}}^{2}}{Q^{2}}+\cdots\right), \\
k_{\mathrm{b}}^{-} & =-\frac{Q}{\sqrt{2}}\left(1-\frac{1-\hat{x}_{\mathrm{N}}\left(1+k_{\mathrm{X}}^{2} / Q^{2}\right)}{1-\hat{x}_{\mathrm{N}}\left(1-q_{\mathrm{T}}^{2} / Q^{2}\right)}\right)=-\frac{\hat{x}_{\mathrm{N}} Q}{\left(1-\hat{x}_{\mathrm{N}}\right) \sqrt{2}}\left(\frac{q_{\mathrm{T}}^{2}}{Q^{2}}+\frac{k_{\mathrm{X}}^{2}}{Q^{2}}+\cdots\right), \\
\mathbf{k}_{\mathrm{T}} & =-\mathbf{q}_{\mathrm{T}}\left(\frac{1-\hat{x}_{\mathrm{N}}\left(1+k_{\mathrm{X}}^{2} / Q^{2}\right)}{1-\hat{x}_{\mathrm{N}}\left(1-q_{\mathrm{T}}^{2} / Q^{2}\right)}\right)=-\mathbf{q}_{\mathrm{T}}\left(1-\frac{\hat{x}_{\mathrm{N}}}{1-\hat{x}_{\mathrm{N}}}\left(\frac{q_{\mathrm{T}}^{2}}{Q^{2}}+\frac{k_{\mathrm{X}}^{2}}{Q^{2}}\right)+\cdots\right) .
\end{aligned}
$$

On the second line, the "..." represents higher powers in an expansion in small $q_{\mathrm{T}}^{2} / Q^{2}$ and $k_{\mathrm{X}}^{2} / Q^{2}$. When $q_{\mathrm{T}}^{2} / Q^{2} \rightarrow 0$ and $k_{\mathrm{X}}^{2} / Q^{2} \rightarrow 0$, the kinematics of the struck parton approach the kinematics of TMD factorization, or the handbag contribution in collinear factorization, with the errors in each component proportional to $q_{\mathrm{T}}^{2} / Q^{2}$.

The most basic of partonic approximations is that the masses and off-shellness of partons is small relative to the hard scale:

$$
k_{\mathrm{i}}^{2} / Q^{2} \rightarrow 0 \quad k_{\mathrm{f}}^{2} / Q^{2} \rightarrow 0 .
$$

On top of these, other approximations are normally needed. For instance, in the current region $k_{\mathrm{f}}$ is aligned with the final state hadron and

$$
k_{\mathrm{f}} \cdot P_{\mathrm{B}} \rightarrow 0
$$

Beyond these, still further approximations apply to different specific partonic subprocesses: 


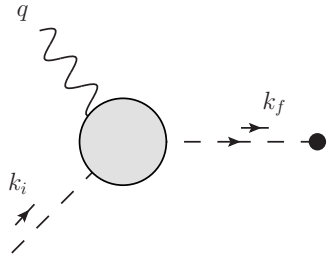

(a)

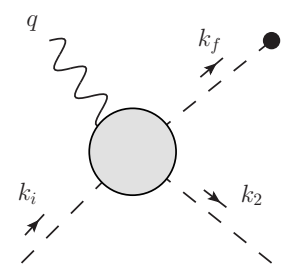

(b)

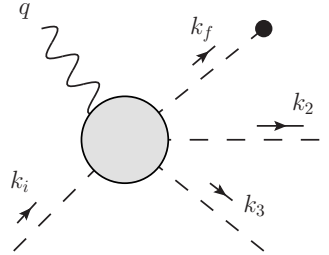

(c)

Figure 6. Examples of hard kinematics. Graph (a) represents handbag kinematics. Graph (b) is $2 \rightarrow 2$ kinematics, which can represent, for instance, the first non-vanishing contribution when we specialize to massless pQCD graphs at large transverse momentum. Graph (c) is $2 \rightarrow 3$ kinematics. We remark that in general, in Graphs (a), (b) and (c) the dashed lines may represent groups of particles, such as those making up a gauge link.

- First, in the $2 \rightarrow 1$ process of figure 6 (a): in order to ensure this kinematical configuration, one assumes that $k_{\mathrm{i}} \rightarrow k$ and drops the $1 / Q^{2}$-suppressed terms in equations like eqs. (4.9)-(4.11).

- Second, for a hard $2 \rightarrow 2$ process shown in figure $6(\mathrm{~b})$ : one needs to have $\left|k^{2}\right| \sim Q^{2}$ while $k_{\mathrm{X}}^{2} / Q^{2} \rightarrow 0$.

- Third, if both $\left|k^{2}\right|$ and $k_{\mathrm{X}}^{2}$ are large, then at least three partons (e.g., figure 6(c)) are ejected at wide angles from the hard collision.

For fixed $x_{\mathrm{N}}, z_{\mathrm{N}}, Q^{2}$, and $\mathbf{P}_{\mathrm{B}, \mathrm{T}}$, only certain $k_{\mathrm{i}}$ and $k_{\mathrm{f}}$ are consistent with any given picture in figure 6 .

\subsection{TMD current region}

Suppose we wish to interpret a particular SIDIS region with a partonic configuration like figure 6(a), corresponding to the current fragmentation region. For a partonic description to hold at all, a minimum requirement is that ratios like eq. (4.12) are very small. So define a ratio

$$
\text { General Hardness Ratio }=R_{0} \equiv \max \left(\left|\frac{k_{\mathrm{i}}^{2}}{Q^{2}}\right|,\left|\frac{k_{\mathrm{f}}^{2}}{Q^{2}}\right|,\left|\frac{\delta k_{\mathrm{T}}^{2}}{Q^{2}}\right|\right) .
$$

and consider regions of $Q$ where $R_{0}$ is less than a certain numerical size for a given set of estimates for $k_{\mathrm{i}}^{2}$ and $k_{\mathrm{f}}^{2}$. Next, since scattering is assumed to be in the current region in figure $6(\mathrm{a})$, the ratio

$$
\text { Collinearity }=R_{1} \equiv \frac{P_{\mathrm{B}} \cdot k_{\mathrm{f}}}{P_{\mathrm{B}} \cdot k_{\mathrm{i}}},
$$

must also be small. See ref. [31] for more discussion $-R_{1}$ corresponds to $R$ from that reference. The expression for $R_{1}$ in terms of rapidities is presented in eq. (E.8).

The $2 \rightarrow 1$ partonic kinematics only apply if $k^{2} / Q^{2} \approx 0$, an approximation that fails if transverse momentum is too large. So define another ratio,

$$
\text { Transverse Hardness Ratio }=R_{2} \equiv \frac{\left|k^{2}\right|}{Q^{2}} .
$$


$R_{2}$ is small for $2 \rightarrow 1$ partonic kinematics. From eq. (4.1),

$$
R_{2}=\left|-\left(1-\hat{z}_{\mathrm{N}}\right)-\hat{z}_{\mathrm{N}} \frac{q_{\mathrm{T}}^{2}}{Q^{2}}-\frac{\left(1-\hat{z}_{\mathrm{N}}\right) k_{\mathrm{f}}^{2}}{Q^{2} \hat{z}_{\mathrm{N}}}-\frac{\delta \mathbf{k}_{\mathrm{T}}^{2}}{\hat{z}_{\mathrm{N}} Q^{2}}+\frac{2 \mathbf{q}_{\mathrm{T}} \cdot \delta \mathbf{k}_{\mathrm{T}}}{Q^{2}}\right| \approx\left(1-\hat{z}_{N}\right)+\hat{z}_{N} \frac{q_{\mathrm{T}}^{2}}{Q^{2}} .
$$

Note that this suggests $q_{\mathrm{T}}$ from eq. (2.21) as the most useful transverse momentum for quantifying transverse momentum hardness relative to $Q$; if $q_{\mathrm{T}}^{2} / Q^{2} \sim 1$, then $R_{2} \sim 1$ for both large and small $\hat{z}_{N}$ while if $q_{\mathrm{T}}^{2} / Q^{2} \ll 1$ and $\zeta \sim z_{\mathrm{N}}$ (as in the current fragmentation region with TMDs) then $R_{2} \ll 1$ (see also discussion in ref. [32]).

If the SIDIS region corresponds to $2 \rightarrow 2$ hard partonic kinematics, then $R_{2}$ must be large $(\sim 1)$. However, then the ratio $k_{\mathrm{X}}^{2} / Q^{2}$ must be small since there is only one unobserved parton, and its invariant mass must be small relative to hard scales to qualify as a single massless parton. (See figure 6(b).) If $k_{2}$ is a massless on-shell quark or gluon, then $k_{2}^{2}=0$ and this places a strong kinematical constraint on relationship between the momentum fractions $\xi$ and $\zeta$. See, for example, eq. (83) of [17]. So define one more ratio,

$$
\text { Spectator Virtuality Ratio }=R_{3} \equiv \frac{\left|k_{\mathrm{X}}^{2}\right|}{Q^{2}} \text {. }
$$

Large $R_{2}$, but small $R_{3}$, corresponds to $2 \rightarrow 2$ parton kinematics. Large $R_{2}$ and large $R_{3}$ corresponds to partonic scattering with three or more final state partons, such as figure $6(\mathrm{c})$.

To see that the size of $R_{2}$, eq. (4.17), reflects the importance of transverse momentum, we repeat an argument very similar to that on page 4 of ref. [32]. Note that Feynman graphs corresponding to the inside of the box in figure 6 contain propagator denominators of the form

$$
\frac{1}{k^{2}+O\left(m^{2}\right)}, \quad \frac{1}{k^{2}+O\left(Q^{2}\right)},
$$

where the denominators with $+O\left(Q^{2}\right)$ arise in corrections to the virtual photon vertex or internal propagators from the emission of wide-angle $k_{\mathrm{X}}$ partons. Note also that $k \cdot q \sim$ $q \cdot P=O\left(Q^{2}\right)$. Possible approximations to these denominators are representative of the approximations needed in derivations of factorization. If $\left|k^{2}\right| \sim Q^{2}$, the $O\left(m^{2}\right)$ terms in the denominators are negligible so that the part of the graph inside the box can be calculated in perturbative QCD using both $Q^{2}$ and $k^{2}$ as equally good hard scales. In this case, and $k_{\mathrm{X}}^{2} \ll Q^{2}$, then figure 6 (b) becomes the relevant picture. However, if $\left|k^{2}\right| \ll Q^{2}$, the $O\left(m^{2}\right)$ terms in the first of the denominators in eq. (4.19) must be kept. Then, a $\left|k^{2}\right| / Q^{2} \ll 1$ approximation in the second denominator can be used, and it is this type of approximation that leads to TMD factorization at small transverse momentum. This is the handbag topology in figure $6(\mathrm{a})$. Note that the $k$ line has become the target parton. Using eq. (4.1) and eq. (4.8) for $k^{2}$ gives eq. (4.17).

\subsection{Hard transverse momentum}

In perturbative QCD, the lowest order (in $O\left(\alpha_{s}\right)$ ) contribution to large transverse momentum is the partonic $2 \rightarrow 2$ process. Again, all partons are massless and on-shell, and the picture is figure $6(\mathrm{~b})$. Since there is only one unobserved massless parton in this region, it correspond to $k_{X}^{2}=0$. To see that it is the ratio $R_{3}$ in eq. (4.18) that must be small in 
this region, consider how the size of $k_{X}^{2}$ affects the denominators in eq. (4.19) at fixed $\hat{x}_{N}$, large $q_{\mathrm{T}}$, and $Q^{2}$ by expressing $\left|k^{2} / Q^{2}\right|$ in terms of $k_{X}^{2}$ instead of $\hat{z}_{N}$ :

$$
\left|\frac{k^{2}}{Q^{2}}\right|=\frac{1}{1-\hat{x}_{N}+\hat{x}_{N} q_{\mathrm{T}}^{2} / Q^{2}}\left[\frac{q_{\mathrm{T}}^{2}}{Q^{2}}+\hat{x}_{N} \frac{k_{X}^{2}}{Q^{2}}\left(1-\frac{q_{\mathrm{T}}^{2}}{Q^{2}}\right)\right] .
$$

To get a simple form, we have already assumed here that $k_{\mathrm{i}}^{2}$ and $k_{\mathrm{f}}^{2}$ are negligible. In propagators, therefore, the size of $k^{2}$ is independent of $k_{X}^{2}$ at large $k_{\mathrm{T}}^{2}$ if $k_{X}^{2} / Q^{2} \ll 1$ and $\hat{x}_{N}$ is not too close to 1 . Otherwise, if $R_{3}$ in eq. (4.18) becomes large, the $2 \rightarrow 3$ or greater cases are likely the more applicable partonic subprocesses. In pQCD this means that $O\left(\alpha_{s}^{2}\right)$ or higher calculations are needed.

Different combinations of sizes for the above ratios correspond to other regions. For example, the target fragmentation region handles cases where $R_{1}$ gets large - see section 4.3 below. All of the approximations discussed above are intertwined in potentially complicated ways, especially when $Q$ is not especially large and mass effects may be non-negligible. This can make even crude, order-of-magnitude estimates of their effects nontrivial, although the influence of model assumptions should diminish rapidly at large $Q$. The catalogue of ratios represented by the $R_{0}-R_{3}$ is meant to make this more straightforward to check.

A choice concerning acceptable ranges of $R_{0}, R_{1}, R_{2}$, and $R_{3}$ translates into a choice about the range of possible reasonable values for the components of $k_{\mathrm{i}}$ and $k_{\mathrm{f}}$. In practice, this might be more conveniently stated in reverse. That is, one starts with general expectations regarding the sizes of the partonic components of $k_{\mathrm{i}}$ and $k_{\mathrm{f}}$ based on models and/or theoretical considerations. The question then becomes whether the resulting $R_{0}, R_{1}, R_{2}$, and $R_{3}$ are consistent with a particular region of partonic kinematics (hard, current region, large transverse momentum, etc).

Our aim here is not to address any particular theoretical framework for estimating intrinsic properties of partons, or to estimate exactly acceptable ranges for the above ratios, but only to demonstrate how, once these choices are made, they fix the relationship between external kinematics and the region of partonic kinematics.

\subsection{Target and soft regions}

If, in contrast to the discussion in section 4.1 and section 4.2 , the hadron is in the target fragmentation region (see figure 7 ), then

$$
P_{\mathrm{B}} \cdot P \ll Q^{2},
$$

In the target region, $z_{\mathrm{h}}$ is no longer as useful for parameterizing the process since it no longer necessarily describes a momentum fraction - see eq. (2.22) and note that the quantity under the square root diverges as $z_{\mathrm{h}} \rightarrow 0$. In terms of $x_{\mathrm{h}}, z_{\mathrm{N}}$ is:

$$
\begin{aligned}
z_{\mathrm{N}} & =\frac{\sqrt{4 x_{\mathrm{Bj}}^{2}\left(M_{\mathrm{B}}^{2} / Q^{2}\right)\left(1-q_{\mathrm{T}}^{2} / Q^{2}\right)+x_{\mathrm{h}}^{2}}-x_{\mathrm{h}}}{2 x_{\mathrm{Bj}}\left(1-q_{\mathrm{T}}^{2} / Q^{2}\right)} \\
& =\frac{M_{\mathrm{B}}^{2} x_{\mathrm{Bj}}}{Q^{2} x_{\mathrm{h}}}-\frac{M_{\mathrm{B}}^{4} x_{\mathrm{Bj}}^{3}\left(Q^{2}-q_{\mathrm{T}}^{2}\right)}{Q^{6} x_{\mathrm{h}}^{3}}+O\left(\frac{M_{\mathrm{B}}^{6}\left(Q^{2}-q_{\mathrm{T}}^{2}\right)^{2}}{Q^{10}}\right),
\end{aligned}
$$




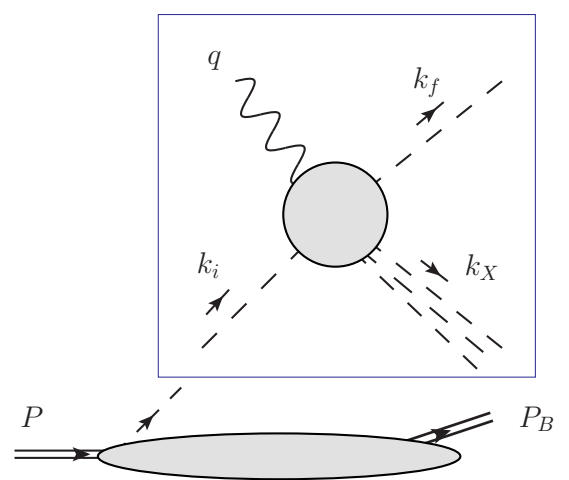

Figure 7. A hadron produced in the target region - see eq. (4.21). Hadrons produced from the hard part are not observed.

where we have kept the solution that gives exactly $z_{\mathrm{N}}=0$ when $P_{\mathrm{B}}$ is exactly massless and collinear to $P$. Now,

$$
\begin{aligned}
P_{\mathrm{B}} \cdot P & =\frac{M M_{\mathrm{B}, \mathrm{T}}}{2}\left(e^{\Delta y}+e^{-\Delta y}\right) \\
& =\frac{M^{2} x_{\mathrm{Bj}}\left(M_{\mathrm{B}}^{2}+q_{\mathrm{T}}^{2} z_{\mathrm{N}}^{2}\right)}{Q z_{\mathrm{N}}\left(\sqrt{4 M^{2} x_{\mathrm{Bj}}^{2}+Q^{2}}+Q\right)}+\frac{Q z_{\mathrm{N}}\left(\sqrt{4 M^{2} x_{\mathrm{Bj}}^{2}+Q^{2}}+Q\right)}{4 x_{\mathrm{Bj}}} .
\end{aligned}
$$

Equation (4.23) is no larger than $O\left(m^{2}\right)$ if $z_{\mathrm{N}} \sim m^{2} / Q^{2}$ and $q_{\mathrm{T}}^{2} z_{\mathrm{N}}^{2} / Q^{2} \ll 1$. So for the target region, eq. (4.21) with eqs. (4.22)-(4.23) means

$$
z_{\mathrm{N}}=\Theta\left(\frac{m^{2}}{Q^{2}}\right) .
$$

The "Big $\Theta$ " symbol is used because the first term in eq. (4.23) puts a lower limit on acceptable sizes for $z_{\mathrm{N}}$. In other words, the target region criterion fails both when $z_{\mathrm{N}} \ll$ $m^{2} / Q^{2}$ as well as when $z_{\mathrm{N}} \gg m^{2} / Q^{2}$. From eq. (4.22), this means the target fragmentation criterion in terms of $x_{\mathrm{h}}, x_{\mathrm{Bj}}$ and $\mathbf{P}_{\mathrm{B}, \mathrm{b}, \mathrm{T}}$ is

$$
\frac{x_{\mathrm{h}}}{x_{\mathrm{Bj}}}=O(1), \quad \frac{q_{\mathrm{T}}^{2} z_{\mathrm{N}}^{2}}{Q^{2}}=\frac{\mathbf{P}_{\mathrm{B}, \mathrm{b}, \mathrm{T}}^{2}}{Q^{2}} \ll 1 .
$$

To translate eq. (4.21) into a dimensionless ratio, define

$$
R_{1}^{\prime}=\frac{P_{\mathrm{B}} \cdot P}{Q^{2}}=\frac{M^{2} x_{\mathrm{Bj}}\left(M_{\mathrm{B}}^{2}+q_{\mathrm{T}}^{2} z_{\mathrm{N}}^{2}\right)}{Q^{3} z_{\mathrm{N}}\left(\sqrt{4 M^{2} x_{\mathrm{Bj}}^{2}+Q^{2}}+Q\right)}+\frac{z_{\mathrm{N}}\left(\sqrt{4 M^{2} x_{\mathrm{Bj}}^{2}+Q^{2}}+Q\right)}{4 x_{\mathrm{Bj}} Q} .
$$

Therefore, the target region criterion is

$$
R_{1}^{\prime} \ll 1 .
$$

In [31], it was $1 / R_{1}$ that was used to characterize the target region, and that is another acceptable definition, but eq. (4.26) has the advantage of working even when $k_{\mathrm{i}}$ differs significantly from $P$ and of being simpler to calculate. 
It is possible that for some hadrons, $R_{2} \ll 1$, while neither $R_{1}$ nor $R_{1}^{\prime}$ is small. We call this the soft region since such hadrons are not a product of hard scattering but do not associate in any obvious way with a quark or target direction. These hadrons may be products of fragmentation of soft quarks and gluons that fill in the central rapidity region between the struck and the outgoing hadrons.

\section{$5 \quad$ Numerical examples}

In section 4 we defined the ratios $R_{1}, R_{2}, R_{3}$ and $R_{1}^{\prime}$ and described how they can help to connect different partonic pictures to specific hadronic kinematics. These quantities can allow in principle to identify kinematical regions where a specific physical picture should be valid, i.e. TMD region section 4.1, hard gluon radiation region section 4.2 , target fragmentation region section 4.3. In this article, we do not attempt to provide a specific demarcation of such regions, but rather establish the (up to now missing) language to perform such analyses. We provide a web tool to enable this type of studies [12]. We stress that a more specific determination of the SIDIS regions requires numerical estimates of the partonic momenta.

It is helpful to sketch the landscape of possible scenarios in a transverse momentum versus rapidity map like the one shown in figure 8 . Each of the regions discussed in section 4 is represented there as a colored blob, and the task is to determine the sizes of the blobs, their borders, and their degree of overlap. The relevant power suppression factors are shown. (Recall, for example, eq. (4.17).)

To give more detailed examples, a few assumptions about non-perturbative properties of partons are necessary: $300 \mathrm{MeV}$ is a typical estimate of non-perturbative mass scales, so we try $k_{\mathrm{i}}=k_{\mathrm{f}}=\delta k_{\mathrm{T}}=300 \mathrm{MeV}$. Also, to start with we assume that $\mathbf{q}_{\mathrm{T}} \cdot \delta \mathbf{k}_{\mathrm{T}}=q_{\mathrm{T}} \delta k_{\mathrm{T}}$. Azimuthal effects may be added later.

In addition, the particular partonic kinematics of interest need to be specified. Say, for example, that the goal is to examine target partons in the valence region (such as discussed on page 3 of ref. [6]). Then the focus should be on momentum fraction values of $\xi$ roughly around 0.3. For $\zeta$, we might reasonably focus on values where collinear fragmentation functions are large but have reasonably small uncertainties, say $\zeta \approx 0.3$. From figure 3 , JLab 12 measurements at $x_{\mathrm{Bj}} \approx 0.2$ may reach to as large as about $2 \mathrm{GeV}$ in $Q$. First let's consider the overall kinematics. The unobserved invariant mass-squared for the SIDIS process reads

$$
\begin{aligned}
& W_{\mathrm{SIDIS}}^{2}=M^{2}+M_{\mathrm{B}}^{2}+\frac{Q^{2}\left(1-x_{\mathrm{Bj}}-z_{\mathrm{h}}\right)}{x_{\mathrm{Bj}}}+\frac{Q^{4} z_{\mathrm{h}}\left(\sqrt{1+\frac{4 M^{2} x_{\mathrm{Bj}}^{2}}{Q^{2}}} \sqrt{1-\frac{4 M^{2} x_{\mathrm{Bj}}^{2} M_{\mathrm{B}, \mathrm{T}}^{2}}{z_{\mathrm{h}}^{2} Q^{4}}}-1\right)}{2 M^{2} x_{\mathrm{Bj}}^{2}} \\
& M, \stackrel{M_{\mathrm{B}} \rightarrow 0}{=} \frac{Q^{2}\left(1-x_{\mathrm{Bj}}\right)\left(1-z_{\mathrm{h}}\right)}{x_{\mathrm{Bj}}}-\frac{\mathbf{P}_{\mathrm{B}, \mathrm{T}}^{2}}{z_{\mathrm{h}}}
\end{aligned}
$$

Contour plots of $W_{\text {SIDIS }}^{2}$, eq. (5.1), are shown for a pion mass in figure 9 for (a) $q_{\mathrm{T}}=0$ and (b) $q_{\mathrm{T}}=2.0 \mathrm{GeV}$, giving a sense of what is kinematically possible for the SIDIS remnant 


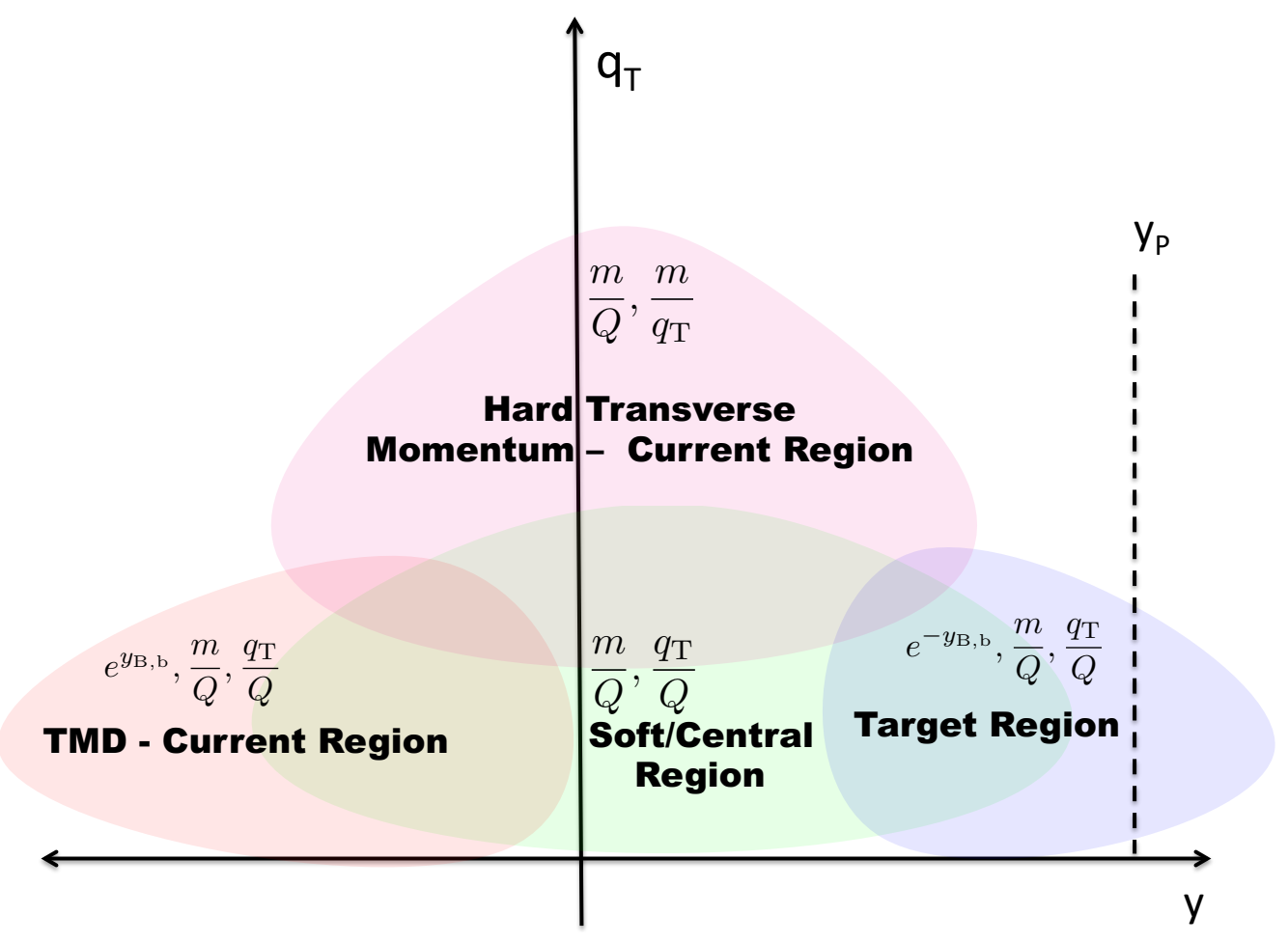

Figure 8. Sketch of kinematical regions of SIDIS in terms of the produced hadron's Breit frame rapidity and transverse momentum. In each region, the type of suppression factors that give factorization are shown. (The exact size and shape of each region may be very different from what is shown and depends on quantities like $Q$ and the hadron masses.) In the Breit frame, according to eq. (E.7), partons in the handbag configuration are centered on $y \approx 0$ if $-k_{\mathrm{i}}^{2} \approx k_{\mathrm{f}}^{2}=O\left(m^{2}\right)$. The shaded regions in the sketch are shifted somewhat toward the target rapidity $y_{P, \mathrm{~b}}$ (the vertical dashed line) to account for the behavior of the rapidities, eq. (E.1), when $z_{\mathrm{N}}$ and $x_{\mathrm{N}}$ are small.

at different $q_{\mathrm{T}}$ and for lower $Q$. The expectation is that the area near the kinematically forbidden region, where the final state phase space vanishes, does not readily separate into distinct regions as in figure 8 . So in the below we will focus on kinematics away from those boundaries. Also, for now we will restrict to large enough $Q$ so that $R_{0}$ in eq. (4.14) is negligible, so $R_{1}$ is the first of the $R_{0}-R_{3}$ that we will consider here.

For the representative values discussed above $\left(\xi=0.3, z_{\mathrm{h}}=0.25, \zeta=0.3\right.$ and a small $\left.q_{\mathrm{T}}=0.3 \mathrm{GeV}\right)$, values of $R_{1}$ are shown on the $Q$ vs. $x_{\mathrm{Bj}}$ contour plot in figure 10 . The trend is as expected: at large $Q$ and not-too-large $x_{\mathrm{Bj}}, R_{1}$ remains small for all transverse momenta, while corrections might be necessary at smaller $Q$ and larger $x_{\mathrm{Bj}}$. In addition to confirming the current-region approximation, which holds valid where collinearity $R_{1}$ is small, it is necessary to map out the applicability of large and small transverse momentum approximations. For this we turn to $R_{2}$. figure 11 is an example that corresponds to the same kinematics as figure 10. It confirms basic expectations, such as that what constitutes "large- $q_{\mathrm{T}}$ " grows with $Q$. It also shows that, while the hadron is in the current region for most $q_{\mathrm{T}}$ as in figure $10(\mathrm{a}, \mathrm{b})$, the small transverse momentum region shown in figure 11 (a) is much more restrictive. For $q_{\mathrm{T}} \lesssim 0.5 \mathrm{GeV}, R_{2}$ is firmly in the small transverse momentum 
(a) $q_{T}=0 \mathrm{GeV} \quad z_{h}=0.25$

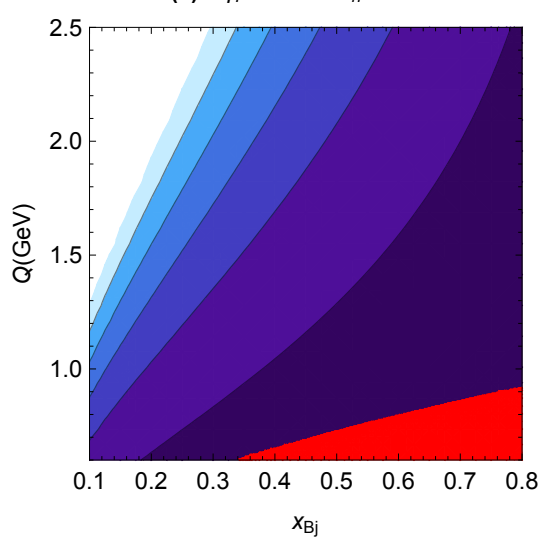

(b) $q_{T}=2 . \mathrm{GeV} \quad z_{h}=0.25$

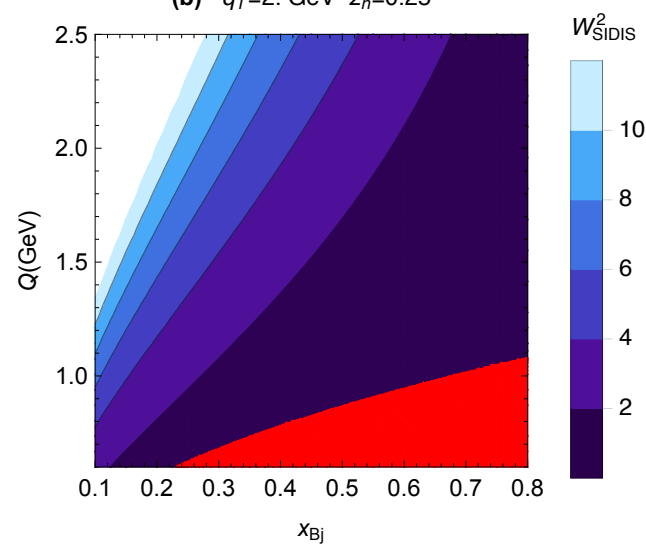

Figure 9. Plots (a)-(b) show $W_{\mathrm{SIDIS}}^{2}$, eq. (5.1), for $q_{\mathrm{T}}=0$ and $q_{\mathrm{T}}=2.0 \mathrm{GeV}$ respectively for the case of a produced pion. Here $z_{\mathrm{h}}=0.25$ in each case. The red region is kinematically forbidden. Near to the kinematically forbidden region, it is to be expected that a clear separation into regions along the lines of figure 8 will break down. The classification according to the sizes of $R_{0}-R_{3}$ is cleaner at larger $Q$ and with small but fixed $x_{\mathrm{Bj}}$. Note that the corresponding plots for a heavier final state hadron have a larger forbidden region.

(a) $M_{B}=0.14 \mathrm{GeV} \quad q_{T}=0.3 \mathrm{GeV}$

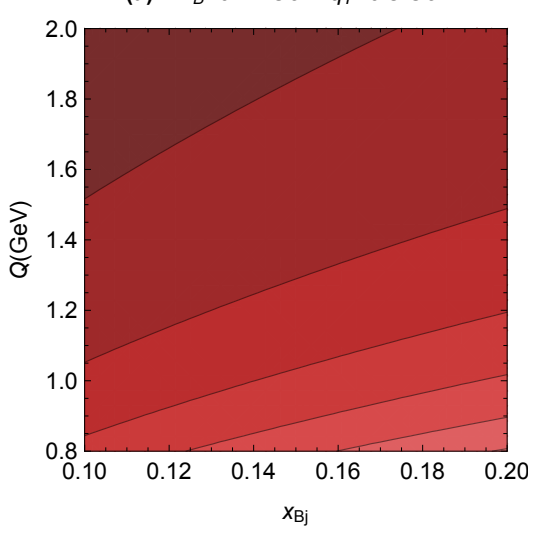

(c) $M_{B}=0.5 \mathrm{GeV} \quad q_{T}=0.3 \mathrm{GeV}$

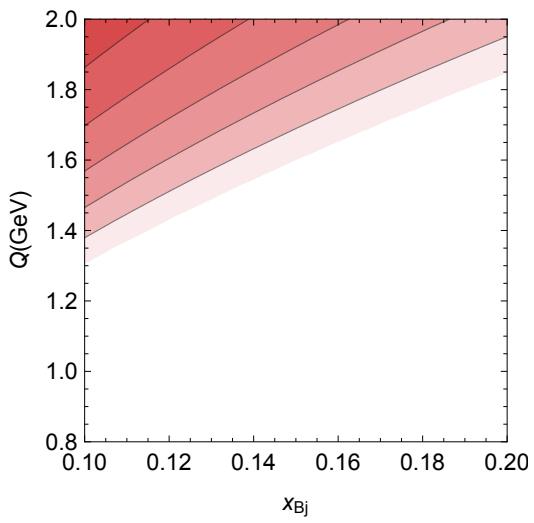

(b) $M_{B}=0.14 \mathrm{GeV} \quad q_{T}=2 . \mathrm{GeV}$

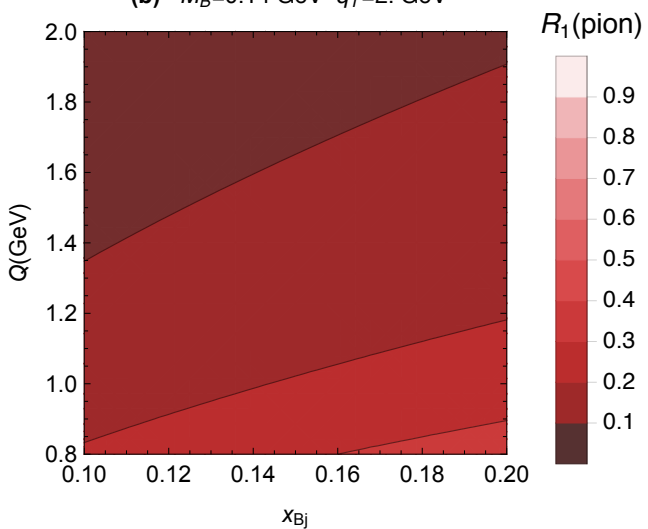

(d) $M_{B}=0.5 \mathrm{GeV} \quad q_{T}=2 . \mathrm{GeV}$

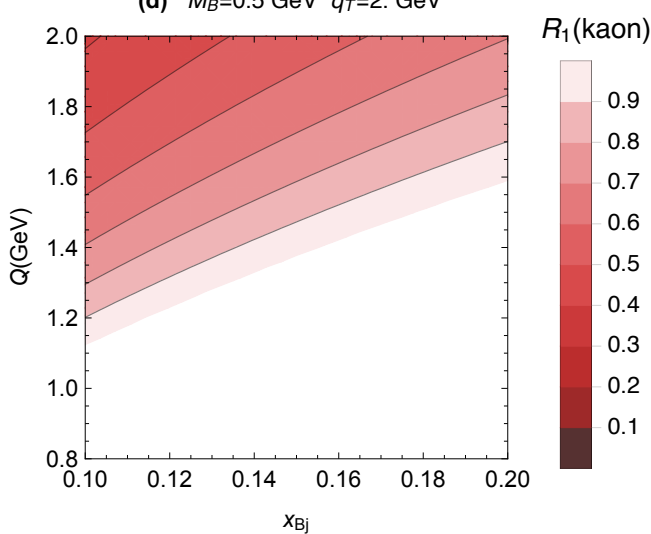

Figure 10. Collinearity $\left(R_{1}\right.$ from eq. (4.15) for fixed $z_{h}=0.25, \zeta=0.3$ and $\xi=0.2$. Top panels show the ratio for $M_{B}=m_{\pi}$ at (a) small transverse momentum $\left(q_{\mathrm{T}}=0.3 \mathrm{GeV}\right)$ and (b) $q_{\mathrm{T}}=2.0 \mathrm{GeV}$. Similar cases for $M_{B}=m_{K}$ are shown in the bottom panels, (c) and (d). 
(a) $M_{B}=0.14 \mathrm{GeV} \quad x_{\mathrm{Bj}}=0.2$

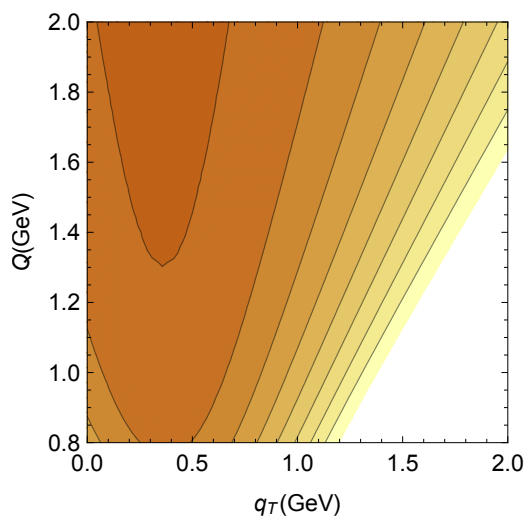

(c) $M_{B}=0.5 \mathrm{GeV} \quad x_{\mathrm{Bj}}=0.2$

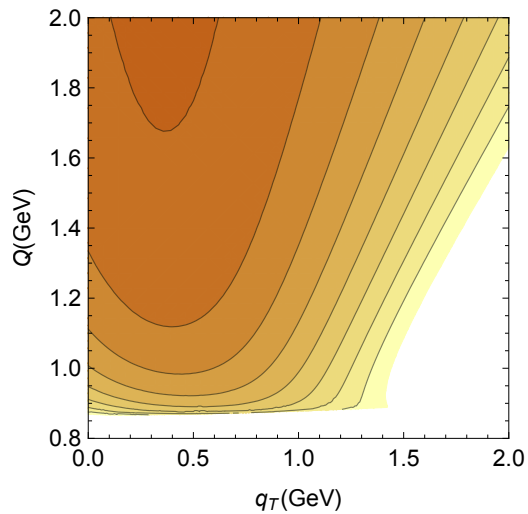

(b) $M_{B}=0.14 \mathrm{GeV} \quad x_{\mathrm{Bj}}=0.01$

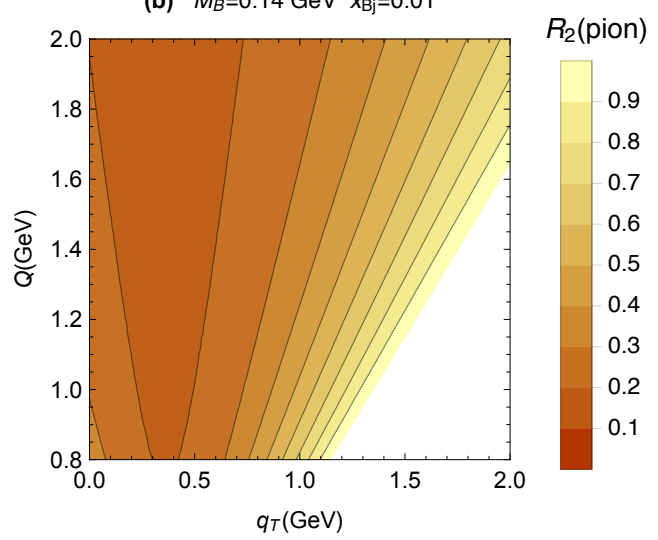

(d) $M_{B}=0.5 \mathrm{GeV} \quad x_{\mathrm{Bj}}=0.01$

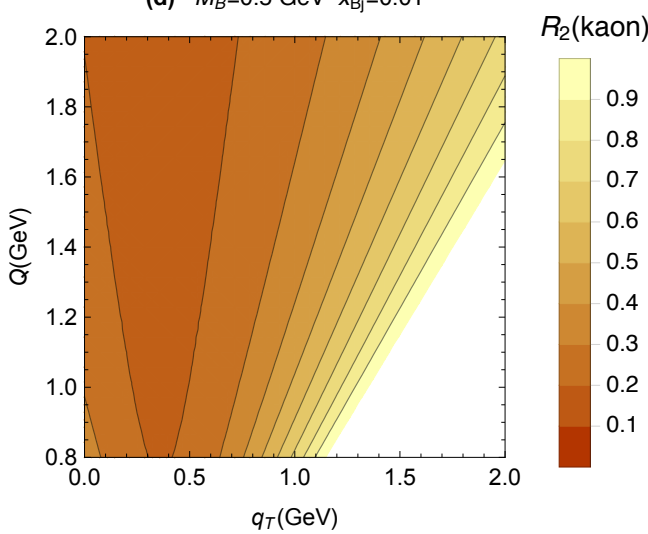

Figure 11. Transverse momentum hardness, $R_{2}$, from eq. (4.16) for fixed $z_{h}=0.25, \zeta=0.3$ and $\xi=0.2$. Top panels show the ratio for $M_{B}=m_{\pi}$ at (a) $x_{\mathrm{Bj}}=0.2$ and (b) $x_{\mathrm{Bj}}=0.01$. Similar cases for $M_{B}=m_{K}$ are shown in the bottom panels, (c) and (d).

region (small values of $R_{2}$ ) for most of the $Q$ shown, while for $q_{\mathrm{T}} \gtrsim 1.5 \mathrm{GeV} R_{2}$ indicates that we are well in the large transverse momentum region.

There is a broad intermediate region where the situation is not clear. The flavor of the final state hadron is a decisive factor in determining the relevant factorization region. For example, comparing the plots of $R_{1}$ in figure 10 for (a) $M_{B}=m_{\pi}$, and (c) $M_{B}=m_{K}$, shows a completely different behaviour of the collinearity ratio $R_{1}$. For $Q=1.5 \mathrm{GeV}$ and $x_{B j}=0.1, R_{1} \approx 0.1$ for pions and $R_{1} \approx 0.8$ for kaons. If $R_{1} \approx 0.8$ is taken to be large, then confidence that one is in the current region deteriorates.

The flavor of the final state hadron has little effect on the transverse momentum hardness, $R_{2}$, from eq. (4.16). From figure 11 (a) and figure 11 (c) flavor dependence is only noticeable at low $\mathrm{Q}$ and even then the effect is small. To summarize, the produced hadron mass affects collinearity $R_{1}$ significantly, but does not appear to be a primary factor in determining transverse hardness $R_{2}$.

Within a specific example, collinearity $R_{1}$ and transverse hardness $R_{2}$ have helped us to map out the current kinematic region (small $R_{1}$ ) and to separate the "small" from the "large" transverse momentum regions (small $R_{2}$ vs large $R_{2}$ ). The small $R_{2}$ will reasonably 


\begin{tabular}{|c|c|c|c|c|c|}
\hline & $R_{0}$ & $R_{1}$ & $R_{2}$ & $R_{3}$ & $R_{1}^{\prime}$ \\
\hline TMD Current region & small & small & small & $\mathrm{X}$ & large \\
Hard region & small & small & large & small (low order pQCD) & large \\
& small & small & large & large (high order pQCD) & large \\
Target region & small & large & $\mathrm{X}$ & $\mathrm{X}$ & small \\
Soft region & small & large & small & $\mathrm{X}$ & large \\
\hline
\end{tabular}

Table 1. Examples for sizes of ratios corresponding to particular regions of SIDIS. The "X" means "irrelevant or ill-defined." This ranking should be viewed as schematic since "small" and "large" need to be defined quantitatively and can in general be scale-dependent.

correspond to a region where we expect TMD factorization to apply, while for the large $R_{2}$ a collinear factorization will be appropriate.

We present a catalogue of typical sizes for ratios corresponding to regions in SIDIS in table 1.

At this stage, one might wonder whether a LO calculation could be enough or whether higher order perturbative corrections are necessary. This is where $R_{3}$ comes into the game: large $R_{3}$ coupled with large $R_{2}$ signal a large $q_{T}$ region where presumably higher order pQCD corrections are relevant, while small $R_{3}$ together with small $R_{2}$ indicate a TMD current region, which requires a TMD factorization scheme.

The behavior of $R_{3}$ is shown in figure 12 as a function of $x_{\mathrm{Bj}}$ and $Q$. Note that at large transverse momentum there is a linear region in the $Q$ versus $x_{\mathrm{Bj}}$ plane where the $2 \rightarrow 2$ process is the optimal description $\left(R_{3}\right.$ is small) and thus low order QCD computations may be applicable.

Clearly the above examples only apply to the specific case we have chosen, corresponding to specific values of the kinematic variables $\left(\xi=0.3, z_{\mathrm{h}}=0.25, \zeta=0.3, q_{\mathrm{T}}=0.3 \mathrm{GeV}\right)$ and of the non perturbative parameters $\left(k_{\mathrm{i}}=k_{\mathrm{f}}=\delta k_{\mathrm{T}}=300 \mathrm{MeV}, \mathbf{q}_{\mathrm{T}} \cdot \delta \mathbf{k}_{\mathrm{T}}=q_{\mathrm{T}} \delta k_{\mathrm{T}}\right)$. A web tool which allows to compute $R_{1}-R_{3}$ for any kinematic configuration can be found in ref. [12].

The ratios defined in this paper allow model assumptions about quantities like, for example, partonic virtualities to be translated into expectations for the applicability of different factorization-based pictures. There are, of course, many possible ways this might be useful in practice.

The most natural application would be to use the $R$ ratios as criteria to help in the selection of the experimental data samples to use in phenomenological studies of SIDIS processes. These studies inevitably rely on models which are only suitable for specific kinematical ranges. It is therefore crucial to have tools, like the $R$ ratios, to relate assumptions about the partonic degrees of freedom to the classification of the SIDIS kinematic regions.

Clearly these types of analyses will depend also on the precision of the existing data to constrain the non-perturbative parameters, but the $R$ ratios can be used to update them as the quantity of data increases. We leave the dedicated study of incorporating the phenomenology of the SIDIS process for future work. 
(a) $M_{B}=0.14 \mathrm{GeV} \quad q_{T}=0.3 \mathrm{GeV}$

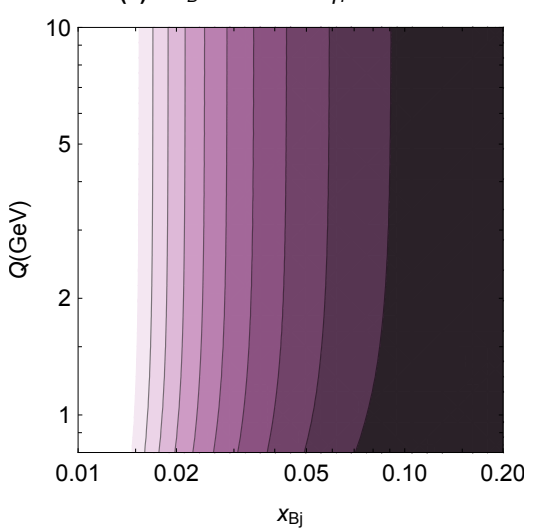

(c) $M_{B}=0.5 \mathrm{GeV} \quad q_{T}=0.3 \mathrm{GeV}$

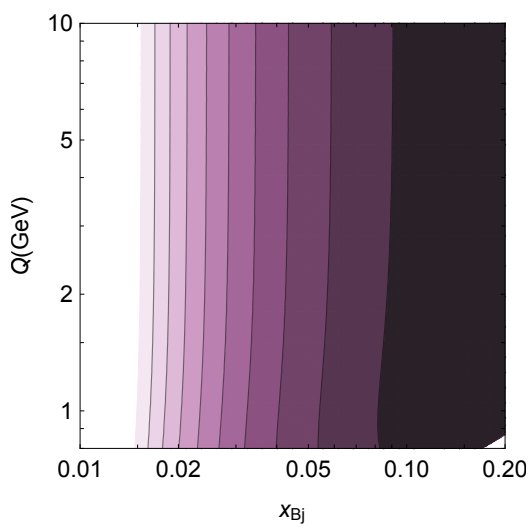

(b) $M_{B}=0.14 \mathrm{GeV} \quad q_{T}=2 . \mathrm{GeV}$

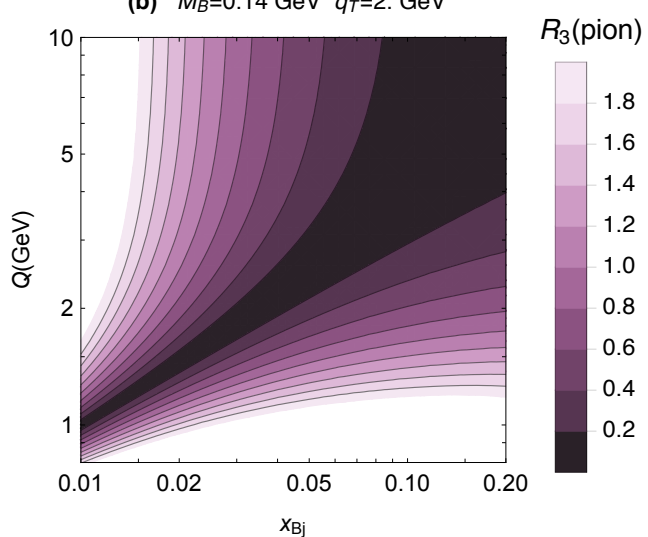

(d) $M_{B}=0.5 \mathrm{GeV} \quad q_{T}=2 . \mathrm{GeV}$

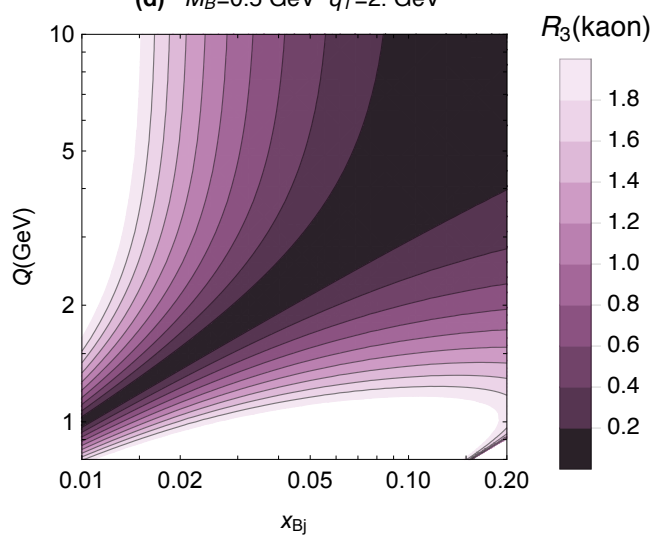

Figure 12. Spectator virtuality ratio, $R_{3}$, from eq. (4.18) for fixed $z_{h}=0.25, \zeta=0.3$ and $\xi=0.2$. Top panels show the ratio for $M_{B}=m_{\pi}$ at (a) small transverse momentum $\left(q_{\mathrm{T}}=0.3 \mathrm{GeV}\right)$ and (b) $q_{\mathrm{T}}=2.0 \mathrm{GeV}$. Similar cases for $M_{B}=m_{K}$ are shown in the bottom panels, (c) and (d). Note that both $x_{\mathrm{Bj}}$ and $Q$ axes are shown in logarithmic scale.

\section{Conclusions}

Since early work in, for example, refs. $[8,15,17]$ there has been a large number of studies on unpolarized SIDIS cross sections [33-39]. Unpolarized SIDIS is, however, only one component in a broad program of phenomenological studies where the universality of parton correlation functions plays a central role in testing pictures of nucleon structure [40-60]. Integrating SIDIS into such a program demands a clear language for identifying kinematical regions with particular underlying partonic pictures, especially in regions of moderate to low $\mathrm{Q}$ where sensitivity to kinematical effects outside the usual very high energy limit becomes non-trivial.

In this paper, we have outlined the ways that the questions about the boundaries between different partonic regimes of SIDIS can be posed systematically, based on the power-law expansions that apply in each region (see figure 8). As the ratios $R_{0}-R_{3}$ described in section 4 show, quantifying the separation between different SIDIS regions requires at least some rough model assumptions for the intrinsic properties of partons. Hence, our position is that region mapping should be viewed as one of the aspects of SIDIS that is to 
be determined with guidance from data, rather than being treated as well-known input. Nevertheless, the $R_{0}-R_{3}$ can already be useful for querying the reasonableness of some region assumptions.

For example, if collinearity $R_{1}$ is found to be approximately 10 for a wide range of even rough models, then a current region assumption could be viewed with skepticism. Conversely, very small values of collinearity $R_{1}$ might be considered a strong signal that one is deep in a regime where a current region fragmentation function picture is appropriate. If, in addition, there is a small transverse hardness ratio $R_{2}$ it may be taken to signal the close proximity to small transverse momentum, where a TMD factorization scheme would be appropriate. If transverse hardness ratio $R_{2}$ and spectator virtuality ratio $R_{3}$ are both large, then high order pQCD corrections are likely important. In a fitting context, the $R_{0^{-}}$ $R_{3}$ can be utilized to fix Bayesian priors. Conversely, the success or failure of theoretical predictions can be used to constrain the ranges of $R_{0}-R_{3}$ that are acceptable for particular regions in future theoretical predictions.

In developing a picture of the likelihood that a particular kinematical region corresponds to a particular partonic picture, one should of course consider a wide range of multiple non-perturbative models for the values of $k_{\mathrm{i}}, k_{\mathrm{f}}$, etc., in addition to sampling from a range of $\zeta, \xi$, and azimuthal angles, and track the values of $R_{0}-R_{3}$, in addition to $x_{\mathrm{N}} / x_{\mathrm{Bj}}$, $z_{\mathrm{N}} / z_{\mathrm{h}}, W_{\mathrm{tot}}^{2}, W_{\text {SIDIS }}^{2}$ to assess the validity of various purely kinematical approximations. The effect of changing quantities like $k_{\mathrm{i}}^{2}$ and $k_{\mathrm{f}}^{2}$ can be examined directly with our web tool ref. [12].

In the future we plan to incorporate this view into phenomenological procedures, particularly in situations with not-too-large $Q$. We hope that this will ultimately contribute to a clearer picture of the borders between different regions and an improved understanding of the transition between hadronic and partonic degrees of freedom.

\section{Acknowledgments}

We thank Eric Moffat, Wally Melnitchouk and Jian-Wei Qiu for useful discussions. We are especially thankful to Markus Diehl for discussions that led to appendix D. T. Rogers, A. Dotson, S. Gordon and N. Sato were supported by the U.S. Department of Energy, Office of Science, Office of Nuclear Physics, under Award Number DE-SC0018106. This work was also supported by the DOE Contract No. DE- AC05-06OR23177, under which Jefferson Science Associates, LLC operates Jefferson Lab. L. Gamberg was supported by the U.S. Department of Energy under grant No. DE-FG02-07ER41460. A. Prokudin was supported by the National Science Foundation under Grant No. PHY-1623454 and the DOE Contract No. DE- AC05-06OR23177, under which Jefferson Science Associates, LLC operates Jefferson Lab. J. O. Gonzalez-Hernandez work was partially supported by Jefferson Science Associates, LLC under U.S. DOE Contract DE-AC05-06OR23177 and by the U.S. DOE Grant DE-FG02-97ER41028. 


\section{A Light-cone variables}

Light-cone variables are defined as follows: for a four-vector $V^{\mu}$,

$$
V^{\mu}=\left(V^{+}, V^{-}, \mathbf{V}_{\mathrm{T}}\right)
$$

where

$$
V^{+}=\frac{V^{0}+V^{z}}{\sqrt{2}}, \quad V^{-}=\frac{V^{0}-V^{z}}{\sqrt{2}}, \quad \mathbf{V}_{\mathrm{T}}=\left(V^{x}, V^{y}\right) .
$$

For a four-momentum $V$, rapidity is defined as usual:

$$
y=\frac{1}{2} \ln \left(\left|\frac{V^{+}}{V^{-}}\right|\right) .
$$

In terms of rapidity, light-cone momentum is:

$$
V=\left(\frac{M_{\mathrm{T}}}{\sqrt{2}} e^{y}, \frac{M_{\mathrm{T}}}{\sqrt{2}} e^{-y}, \mathbf{V}_{\mathrm{T}}\right),
$$

where $V^{2}=M^{2}$ and transverse mass is

$$
M_{\mathrm{T}}=\sqrt{\left|M^{2}+\mathbf{V}_{\mathrm{T}}^{2}\right|} .
$$

For a virtual momentum, $M^{2}<0$ and either the plus or minus light-cone component is negative, e.g.,

$$
V=\left(\frac{M_{\mathrm{T}}}{\sqrt{2}} e^{y},-\frac{M_{\mathrm{T}}}{\sqrt{2}} e^{-y}, \mathbf{V}_{\mathrm{T}}\right) .
$$

In labeling a four-momentum component of $V$, we will write:

$$
V_{\mathrm{b}, \mathrm{c}}^{\mathrm{a}}
$$

where $a$ is the contravariant component, $c$ specifies the reference frame, and $b$ is any other necessary subscript depending on the given context. A two-dimensional transverse momentum is

$$
\mathbf{V}_{\mathrm{b}, \mathrm{c}, \mathrm{T}} \cdot
$$

The frame subscripts $b, c$ on a four-momentum indicate in which frame its components will be expressed.

\section{B Lorentz transformations}

It is often useful to switch back and forth between the photon (e.g., Breit) and hadron frames. For this, define

$$
\kappa \equiv \sqrt{z_{\mathrm{N}}^{2} \mathbf{q}_{\mathrm{T}}^{2}+\frac{M^{2} x_{\mathrm{N}}^{2}\left(M_{\mathrm{B}}^{2}+\mathbf{q}_{\mathrm{T}}^{2} z_{\mathrm{N}}^{2}-\frac{Q^{4} z_{\mathrm{N}}^{2}}{M^{2} x_{\mathrm{N}}^{2}}\right)^{2}}{4 Q^{4} z_{\mathrm{N}}^{2}}}=O\left(\frac{Q^{2}}{m}\right) .
$$


The law to transform a vector $V$ from the Breit frame to the hadron frame is then ${ }^{7}$

$$
\begin{aligned}
V_{\mathrm{H}}^{+}= & \frac{1}{2 M^{2} x_{\mathrm{N}}^{2}}\left(M^{2} x_{\mathrm{N}}^{2}\left(1+\sqrt{1-\frac{z_{\mathrm{N}}^{2} \mathbf{q}_{\mathrm{T}}^{2}}{\kappa^{2}}}\right) V_{\mathrm{b}}^{+}-Q^{2}\left(-1+\sqrt{1-\frac{z_{\mathrm{N}}^{2} \mathbf{q}_{\mathrm{T}}^{2}}{\kappa^{2}}}\right) V_{\mathrm{b}}^{-}\right) \\
& +\frac{Q z_{\mathrm{N}}}{\sqrt{2} M x_{\mathrm{N}} \kappa} \mathbf{q}_{\mathrm{T}} \cdot \mathbf{V}_{\mathrm{b}, \mathrm{T}}, \\
V_{\mathrm{H}}^{-}= & -\frac{1}{2 Q^{2}}\left(M^{2} x_{\mathrm{N}}^{2}\left(-1+\sqrt{1-\frac{z_{\mathrm{N}}^{2} \mathbf{q}_{\mathrm{T}}^{2}}{\kappa^{2}}}\right) V_{\mathrm{b}}^{+}-Q^{2}\left(1+\sqrt{1-\frac{z_{\mathrm{N}}^{2} \mathbf{q}_{\mathrm{T}}^{2}}{\kappa^{2}}}\right) V_{\mathrm{b}}^{-}\right) \\
& -\frac{M x_{\mathrm{N}} z_{\mathrm{N}}}{\sqrt{2} Q \kappa} \mathbf{q}_{\mathrm{T}} \cdot \mathbf{V}_{\mathrm{b}, \mathrm{T}}, \\
\mathbf{V}_{\mathrm{H}, \mathrm{T}}= & \mathbf{V}_{\mathrm{b}, \mathrm{T}} \sqrt{1-\frac{z_{\mathrm{N}}^{2} \mathbf{q}_{\mathrm{T}}^{2}}{\kappa^{2}}}+\mathbf{q}_{\mathrm{T}} \frac{z_{\mathrm{N}}\left(Q^{2} V_{\mathrm{b}}^{-}-M^{2} x_{\mathrm{N}}^{2} V_{\mathrm{b}}^{+}\right)}{\sqrt{2} M Q x_{\mathrm{N}} \kappa}
\end{aligned}
$$

Varying conventions. For us, the hadron frame has zero transverse momentum for the produced hadron and non-zero transverse momentum for the virtual photon $[11,16]$. Note that this is opposite the situation in the hadron frame of Meng-Olness-Soper (MOS) ref. [13]. The MOS hadron frame corresponds to the photon frame of Collins [11]. MOS define a Lorentz invariant four-vector ([13, eq. (10)]) that measures the deviation from the back-to-back configuration. From [13, eq. (11)] and [11, eq. (13.104)], the ref. [11] hadron frame $\mathbf{q}_{\mathrm{H}, \mathrm{T}}^{2}$ is the same as the MOS $\mathbf{q}_{\mathrm{H}, \mathrm{T}}^{2}$ if hadron masses are neglected.

Restricting to the MOS hadron frame, MOS use [13, eq. (11)] and [13, eq. (13)] and $P_{\mathrm{B}}^{2}=0$ to find [13, eq. (12)], which in light-cone coordinates is eq. (2.19) with $M_{\mathrm{B}}^{2}=0$ and with the MOS $\mathbf{q}_{\mathrm{T}}$ defined to point along the positive $x$-axis. In the MOS hadron frame, the transverse part of $P_{\mathrm{B}}$ is always in the $x$ direction and is always positive.

Mulders and Tangerman [16, eqs. (15)-(17)] give general expressions for four vector components that include the effects of hadron masses, and the reference frames used correspond to the hadron and/or photon frames defined above. References such as [20, 24, 61] specialize the photon frame to the target rest frame rather than the Breit frame. $\mathbf{P}_{\mathrm{B}, \mathrm{b}, \mathrm{T}}$ is invariant, however, with respect to boosts along the $z$-axis. Other conventions use some combination of the above. Refs. [62-64] use a hadronic tensor with an extra $1 / 4 z_{\mathrm{h}}$ relative to the above and refs. $[16,18]$ have an extra $1 / 2 M$. The notation of ref. [33] is similar to ref. [13].

\section{Cross sections and structure functions}

Here we establish our conventions for the SIDIS and DIS cross sections. A cross section differential in $N$ final state particles for particle $A$ scattering from particle $B$ is related to

\footnotetext{
${ }^{7}$ The simplest sequence of transformations to get this are: 1) boost from the Breit frame to the proton rest frame 2) rotate until the momentum of the final state hadron is along the negative $z$-axis 3 ) boost along the $z$-axis to a frame where the proton has a light-cone plus component equal to that of Breit frame $P_{\mathrm{b}}^{+}=Q / x_{\mathrm{N}} \sqrt{2}$.
} 
modulus-squared matrix elements $|M|^{2}$ in the usual way:

$$
\begin{aligned}
\mathrm{d} \sigma= & \frac{\left|M^{A, B \rightarrow N}\right|^{2}}{2 \lambda\left(s, m_{A}^{2}, m_{B}^{2}\right)^{1 / 2}} \times \frac{\mathrm{d}^{3} \boldsymbol{p}_{1}}{(2 \pi)^{3} 2 E_{1}} \times \frac{\mathrm{d}^{3} \boldsymbol{p}_{2}}{(2 \pi)^{3} 2 E_{2}} \times \cdots \times \frac{\mathrm{d}^{3} \boldsymbol{p}_{N}}{(2 \pi)^{3} 2 E_{N}} \\
& \times(2 \pi)^{4} \delta^{(4)}\left(k_{A}+k_{B}-\sum_{i=1}^{N} p_{i}\right)
\end{aligned}
$$

with the triangle function

$$
\lambda\left(s, m_{A}^{2}, m_{B}^{2}\right) \equiv s^{2}+m_{A}^{4}+m_{B}^{4}-2 s m_{A}^{2}-2 s m_{B}^{2}-2 m_{A}^{2} m_{B}^{2} .
$$

The usual structure function decompositions on $W_{\text {tot }}^{\mu \nu}$ and $W_{\text {SIDIS }}^{\mu \nu}$ are

$$
\begin{aligned}
W_{\mathrm{tot}}^{\mu \nu}= & \left(-g^{\mu \nu}+\frac{q^{\mu} q^{\nu}}{q^{2}}\right) F_{1}^{\mathrm{tot}}\left(x_{\mathrm{Bj}}, Q^{2}\right)+\frac{\left(P^{\mu}-q^{\mu} \frac{P \cdot q}{q^{2}}\right)\left(P^{\nu}-q^{\nu} \frac{P \cdot q}{q^{2}}\right)}{P \cdot q} F_{2}^{\mathrm{tot}}\left(x_{\mathrm{Bj}}, Q^{2}\right) \\
& + \text { Pol. Dep. } \\
W_{\text {SIDIS }}^{\mu \nu}= & \left(-g^{\mu \nu}+\frac{q^{\mu} q^{\nu}}{q^{2}}\right) F_{1}\left(x_{\mathrm{Bj}}, Q^{2}, z_{\mathrm{h}}, \mathbf{P}_{\mathrm{B}, \mathrm{b}, \mathrm{T}}\right) \\
& +\frac{\left(P^{\mu}-q^{\mu} \frac{P \cdot q}{q^{2}}\right)\left(P^{\nu}-q^{\nu} \frac{P \cdot q}{q^{2}}\right)}{P \cdot q} F_{2}\left(x_{\mathrm{Bj}}, Q^{2}, z_{\mathrm{h}}, \mathbf{P}_{\mathrm{B}, \mathrm{b}, \mathrm{T}}\right)+\text { Pol. Dep. }
\end{aligned}
$$

"Pol. Dep." is a place holder for polarization and azimuthal angle dependent terms, which we leave unspecified for now. The structure functions' explicit dependence on $M$ and $M_{\mathrm{B}}$ has been dropped for brevity. While $x_{\mathrm{Bj}}$ and $z_{\mathrm{h}}$ are shown as the independent variables for the structure functions, it is useful to view them as being themselves functions of $x_{\mathrm{N}}$, $z_{\mathrm{N}}, M$ and $M_{\mathrm{B}}$. We have not done this here in order to avoid over-complicating notations, but it is useful for making kinematical approximations clear, as discussed in [23]. The differential SIDIS cross section in the Breit frame (or any photon frame) is then

$$
\begin{aligned}
\frac{\mathrm{d} \sigma}{\mathrm{d} x_{\mathrm{Bj}} \mathrm{d} y \mathrm{~d} \psi \mathrm{d} z_{\mathrm{N}} \mathrm{d}^{2} \mathbf{P}_{\mathrm{B}, \mathrm{b}, \mathrm{T}}}=\frac{\alpha_{\mathrm{em}}^{2} y}{4 Q^{4} z_{\mathrm{N}}} L_{\mu \nu} W_{\mathrm{SIDIS}}^{\mu \nu} \\
=\frac{\alpha_{\mathrm{em}}^{2}}{2 x_{\mathrm{Bj}} y z_{\mathrm{N}} Q^{2}}\left[\left(1-y-\frac{x_{\mathrm{Bj}}^{2} y^{2} M^{2}}{Q^{2}}\right) F_{2}+y^{2} x_{\mathrm{Bj}} F_{1}+\text { Pol. Dep. }\right] \\
=\frac{\alpha_{\mathrm{em}}^{2}}{4 x_{\mathrm{Bj}} z_{\mathrm{N}} y Q^{2}}\left[\left(1+(1-y)^{2}+\frac{2 x_{\mathrm{Bj}}^{2} y^{2} M^{2}}{Q^{2}}\right) F_{2}-y^{2} F_{L}+\text { Pol. Dep. }\right] \\
=\frac{\alpha_{\mathrm{em}}^{2} y}{4 x_{\mathrm{Bj}} z_{\mathrm{N}} Q^{2}(1-\varepsilon)}\left[F_{T}+\varepsilon F_{L}+\text { Pol. Dep. }\right] .
\end{aligned}
$$

In the last two lines

$$
\begin{aligned}
& F_{T} \equiv 2 x_{\mathrm{Bj}} F_{1}, \\
& F_{L} \equiv\left(1+\frac{4 M^{2} x_{\mathrm{Bj}}^{2}}{Q^{2}}\right) F_{2}-2 x_{\mathrm{Bj}} F_{1}=\left(1+\frac{4 M^{2} x_{\mathrm{Bj}}^{2}}{Q^{2}}\right) F_{2}-F_{T},
\end{aligned}
$$


which are definitions generalized from the inclusive case to SIDIS. To match with other common notational conventions, we have used

$$
\gamma \equiv \frac{2 M x_{\mathrm{Bj}}}{Q}, \quad \varepsilon \equiv \frac{1-y-\frac{\gamma^{2} y^{2}}{4}}{1-y+\frac{y^{2}}{2}+\frac{\gamma^{2} y^{2}}{4}},
$$

along with the identities (see [20, eqs. (2.8)-(2.13)]),

$$
\frac{1-y+\frac{y^{2}}{2}+\frac{y^{2} \gamma^{2}}{4}}{1+\gamma^{2}}=\frac{y^{2}}{2(1-\varepsilon)}, \quad \frac{1-y-\frac{y^{2} \gamma^{2}}{4}}{1+\gamma^{2}}=\frac{y^{2} \varepsilon}{2(1-\varepsilon)} .
$$

A convenient recipe for calculating structure functions is to contract with Lorentz covariant extraction tensors, $\mathrm{P}_{\Gamma}^{\mu \nu}$, defined as

$$
\mathrm{P}_{g}^{\mu \nu}=g^{\mu \nu}, \quad \mathrm{P}_{P P}^{\mu \nu}=P^{\mu} P^{\nu}
$$

Then

$$
F_{1}\left(x_{\mathrm{Bj}}, Q^{2}, z_{\mathrm{h}}, \mathbf{P}_{\mathrm{B}, \mathrm{b}, \mathrm{T}}\right)=\mathrm{P}_{1}^{\mu \nu} W_{\mu \nu, \mathrm{SIDIS}} \quad F_{2}\left(x_{\mathrm{Bj}}, Q^{2}, z_{\mathrm{h}}, \mathbf{P}_{\mathrm{B}, \mathrm{b}, \mathrm{T}}\right)=\mathrm{P}_{2}^{\mu \nu} W_{\mu \nu, \mathrm{SIDIS}},
$$

where

$$
\begin{aligned}
\mathrm{P}_{1}^{\mu \nu} & \equiv-\frac{1}{2} \mathrm{P}_{g}^{\mu \nu}+\frac{2 Q^{2} x_{\mathrm{N}}^{2}}{\left(M^{2} x_{\mathrm{N}}^{2}+Q^{2}\right)^{2}} \mathrm{P}_{P P}^{\mu \nu}=-\frac{1}{2} \mathrm{P}_{g}^{\mu \nu}+\frac{2 x_{\mathrm{Bj}}^{2}}{Q^{2}} \mathrm{P}_{P P}^{\mu \nu}+O\left(\frac{m^{2}}{Q^{2}}\right) \\
\mathrm{P}_{2}^{\mu \nu} & \equiv \frac{12 Q^{4} x_{\mathrm{N}}^{3}\left(Q^{2}-M^{2} x_{\mathrm{N}}^{2}\right)}{\left(Q^{2}+M^{2} x_{\mathrm{N}}^{2}\right)^{4}}\left(\mathrm{P}_{P P}^{\mu \nu}-\frac{\left(M^{2} x_{\mathrm{N}}^{2}+Q^{2}\right)^{2}}{12 Q^{2} x_{\mathrm{N}}^{2}} \mathrm{P}_{g}^{\mu \nu}\right) \\
& =\frac{12 x_{\mathrm{Bj}}^{3}}{Q^{2}} \mathrm{P}_{P P}^{\mu \nu}-x_{\mathrm{Bj}} \mathrm{P}_{g}^{\mu \nu}+O\left(\frac{m^{2}}{Q^{2}}\right)
\end{aligned}
$$

The unobserved invariant mass-squared in inclusive DIS is

$$
W_{\mathrm{tot}}^{2}=M^{2}+\frac{Q^{2}\left(1-x_{\mathrm{Bj}}\right)}{x_{\mathrm{Bj}}} .
$$

In SIDIS it is

$$
\begin{gathered}
W_{\mathrm{SIDIS}}^{2}=M^{2}+M_{\mathrm{B}}^{2}+\frac{Q^{2}\left(1-x_{\mathrm{Bj}}-z_{\mathrm{h}}\right)}{x_{\mathrm{Bj}}}+\frac{Q^{4} z_{\mathrm{h}}\left(\sqrt{1+\frac{4 M^{2} x_{\mathrm{Bj}}^{2}}{Q^{2}}} \sqrt{1-\frac{4 M^{2} x_{\mathrm{Bj}}^{2} M_{\mathrm{B}, \mathrm{T}}^{2}}{z_{\mathrm{h}}^{2} Q^{4}}}-1\right)}{2 M^{2} x_{\mathrm{Bj}}^{2}} \\
M, \stackrel{M_{\mathrm{B}}}{=} \frac{Q^{2}\left(1-x_{\mathrm{Bj}}\right)\left(1-z_{\mathrm{h}}\right)}{x_{\mathrm{Bj}}}-\frac{\mathbf{P}_{\mathrm{B}, \mathrm{T}}^{2}}{z_{\mathrm{h}}} .
\end{gathered}
$$

Note that if both $z_{\mathrm{h}}$ and $x_{\mathrm{Bj}}$ are close to 1 , then $\left|\mathbf{P}_{\mathrm{B}, \mathrm{T}}\right|$ cannot be much greater than zero without hitting the resonance region of $W_{\text {SIDIS }}^{2} \approx 0$. 


\section{Sum rule}

In the appendix, we will work in the target hadron rest frame (a photon frame). Start with the elementary relation

$$
\sum_{B} \int \mathrm{d}^{2} \mathbf{P}_{\mathrm{B}, \gamma, \mathrm{T}} \mathrm{d} z_{\mathrm{N}}\left(\frac{\mathrm{d} \sigma^{B}}{\mathrm{~d} x_{\mathrm{Bj}} \mathrm{d} y \mathrm{~d} \psi \mathrm{d}^{2} \mathbf{P}_{\mathrm{B}, \gamma, \mathrm{T}} \mathrm{d} z_{\mathrm{N}}}\right)=\langle N\rangle \frac{\mathrm{d} \sigma^{\mathrm{tot}}}{\mathrm{d} x_{\mathrm{Bj}} \mathrm{d} y \mathrm{~d} \psi},
$$

where $\langle N\rangle$ is the average multiplicity. Change the $z_{\mathrm{N}}$ variable on the left side to $z_{\mathrm{h}}$. The $\mathrm{d} z_{\mathrm{h}}$ appears in both the integral and the derivative and Jacobian factors cancel:

$$
\sum_{B} \int \mathrm{d}^{2} \mathbf{P}_{\mathrm{B}, \gamma, \mathrm{T}} \mathrm{d} z_{\mathrm{h}}\left(\frac{\mathrm{d} \sigma^{B}}{\mathrm{~d} x_{\mathrm{Bj}} \mathrm{d} y \mathrm{~d} \psi \mathrm{d}^{2} \mathbf{P}_{\mathrm{B}, \gamma, \mathrm{T}} \mathrm{d} z_{\mathrm{h}}}\right)=\langle N\rangle \frac{\mathrm{d} \sigma^{\text {tot }}}{\mathrm{d} x_{\mathrm{Bj}} \mathrm{d} y \mathrm{~d} \psi} .
$$

Expressed in differential form, and for one particular hadron type $B$, this is

$$
\mathrm{d}^{2} \mathbf{P}_{\mathrm{B}, \gamma, \mathrm{T}} \mathrm{d} z_{\mathrm{h}}\left(\frac{\mathrm{d} \sigma^{B}}{\mathrm{~d} x_{\mathrm{Bj}} \mathrm{d} y \mathrm{~d} \psi \mathrm{d}^{2} \mathbf{P}_{\mathrm{B}, \gamma, \mathrm{T}} \mathrm{d} z_{\mathrm{h}}}\right)=\mathrm{d}\left\langle N_{B}\right\rangle \frac{\mathrm{d} \sigma^{\text {tot }}}{\mathrm{d} x_{\mathrm{Bj}} \mathrm{d} y \mathrm{~d} \psi},
$$

where $\mathrm{d}\left\langle N_{B}\right\rangle$ is the number of particles of type $B$ in the differential volume $\mathrm{d}^{2} \mathbf{P}_{\mathrm{B}, \gamma, \mathrm{T}} \mathrm{d} z_{\mathrm{h}}$. Let $E_{B}$ be the energy per particle of type $B$ (in the target rest frame), and multiply both sides of eq. (D.3) by $E_{B}$ :

$$
\begin{aligned}
E_{B} \mathrm{~d}^{2} \mathbf{P}_{\mathrm{B}, \gamma, \mathrm{T}} \mathrm{d} z_{\mathrm{h}}\left(\frac{\mathrm{d} \sigma^{B}}{\mathrm{~d} x_{\mathrm{Bj}} \mathrm{d} y \mathrm{~d} \psi \mathrm{d}^{2} \mathbf{P}_{\mathrm{B}, \gamma, \mathrm{T}} \mathrm{d} z_{\mathrm{h}}}\right) & =E_{B} \mathrm{~d}\left\langle N_{B}\right\rangle \frac{\mathrm{d} \sigma^{\text {tot }}}{\mathrm{d} x_{\mathrm{Bj}} \mathrm{d} y \mathrm{~d} \psi} \\
& =\mathrm{d}\left\langle E_{B}^{\text {all }}\right\rangle \frac{\mathrm{d} \sigma^{\text {tot }}}{\mathrm{d} x_{\mathrm{Bj}} \mathrm{d} y \mathrm{~d} \psi} .
\end{aligned}
$$

$E_{B} \mathrm{~d}\left\langle N_{B}\right\rangle$ is the energy per $B$-particle times the number of $B$ particle in the differential volume, so it is the total energy of all $B$-particles in the differential volume. Therefore, we have defined it as $\mathrm{d}\left\langle E_{B}^{\text {all }}\right\rangle$ in the last equality. Integrating it and summing over all types of final state particles produces the total energy of the entire final state:

$$
\sum_{B} \int \mathrm{d}\left\langle E_{B}^{\text {all }}\right\rangle=E^{\text {tot }}
$$

Note that the sum over $B$ is a sum over all types of particles, not a sum over actual particles. Divide both sides of eq. (D.4) by $q^{0}$ :

$$
\frac{E_{B}}{q^{0}} \mathrm{~d}^{2} \mathbf{P}_{\mathrm{B}, \gamma, \mathrm{T}} \mathrm{d} z_{\mathrm{h}}\left(\frac{\mathrm{d} \sigma^{B}}{\mathrm{~d} x_{\mathrm{Bj}} \mathrm{d} y \mathrm{~d} \psi \mathrm{d}^{2} \mathbf{P}_{\mathrm{B}, \gamma, \mathrm{T}} \mathrm{d} z_{\mathrm{h}}}\right)=\frac{1}{q^{0}} \mathrm{~d}\left\langle E_{B}^{\text {all }}\right\rangle \frac{\mathrm{d} \sigma^{\text {tot }}}{\mathrm{d} x_{\mathrm{Bj}} \mathrm{d} y \mathrm{~d} \psi} .
$$

Integrate over both sides, restore the sum over particle types $B$, and use eq. (D.5) for the right side:

$$
\sum_{B} \int \frac{E_{B}}{q^{0}} \mathrm{~d}^{2} \mathbf{P}_{\mathrm{B}, \gamma, \mathrm{T}} \mathrm{d} z_{\mathrm{h}}\left(\frac{\mathrm{d} \sigma^{B}}{\mathrm{~d} x_{\mathrm{Bj}} \mathrm{d} y \mathrm{~d} \psi \mathrm{d}^{2} \mathbf{P}_{\mathrm{B}, \gamma, \mathrm{T}} \mathrm{d} z_{\mathrm{h}}}\right)=\frac{E^{\mathrm{tot}}}{q^{0}} \frac{\mathrm{d} \sigma^{\text {tot }}}{\mathrm{d} x_{\mathrm{Bj}} \mathrm{d} y \mathrm{~d} \psi} .
$$


Now, in the target rest frame,

$$
z_{\mathrm{h}}=\frac{P \cdot P_{\mathrm{B}}}{P \cdot q}=\frac{E_{B}}{q^{0}}
$$

Also,

$$
q^{0}=\frac{Q^{2}}{2 M x_{\mathrm{Bj}}}, \quad P^{0}=M
$$

From energy conservation,

$$
E^{\text {tot }}=q^{0}+P^{0}
$$

so

$$
\frac{E^{\mathrm{tot}}}{q^{0}}=\frac{q^{0}+P^{0}}{q^{0}}=1+2 x_{\mathrm{Bj}} M^{2} / Q^{2}=\left(1+\frac{\gamma^{2}}{2 x_{\mathrm{Bj}}}\right) .
$$

So, eq. (D.7) becomes

$$
\sum_{B} \int z_{\mathrm{h}} \mathrm{d}^{2} \mathbf{P}_{\mathrm{B}, \gamma, \mathrm{T}} \mathrm{d} z_{\mathrm{h}}\left(\frac{\mathrm{d} \sigma^{B}}{\mathrm{~d} x_{\mathrm{Bj}} \mathrm{d} y \mathrm{~d} \psi \mathrm{d}^{2} \mathbf{P}_{\mathrm{B}, \gamma, \mathrm{T}} \mathrm{d} z_{\mathrm{h}}}\right)=\left(1+\frac{\gamma^{2}}{2 x_{\mathrm{Bj}}}\right) \frac{\mathrm{d} \sigma^{\text {tot }}}{\mathrm{d} x_{\mathrm{Bj}} \mathrm{d} y \mathrm{~d} \psi} .
$$

Now we need to use this to relate the SIDIS and the total DIS structure functions. For the total DIS cross section, the structure function decomposition with standard notational conventions uses eq. (2.30), eq. (2.32), and eq. (C.2). The cross section is thus

$$
\frac{\mathrm{d} \sigma^{\mathrm{tot}}}{\mathrm{d} x_{\mathrm{Bj}} \mathrm{d} y \mathrm{~d} \psi}=\frac{\alpha_{\mathrm{em}}^{2} y}{x_{\mathrm{Bj}} Q^{2}(1-\varepsilon)}\left[F_{T}^{\mathrm{tot}}+\varepsilon F_{L}^{\mathrm{tot}}\right] .
$$

Substituting eq. (D.13) into the right side of eq. (D.12), and substituting eq. (2.37) into the left side gives

$$
\int \mathrm{d} z_{\mathrm{h}} \mathrm{d}^{2} \mathbf{P}_{\mathrm{B}, b, \mathrm{~T}} z_{\mathrm{h}} \frac{1}{4 z_{\mathrm{N}}} \frac{x_{\mathrm{N}}\left(\sqrt{1-\frac{4 M^{2} x_{\mathrm{Bj}}^{2} M_{\mathrm{B}, \mathrm{T}}^{2}}{Q^{4} z_{\mathrm{h}}^{2}}}+1\right)}{2 x_{\mathrm{Bj}} \sqrt{1-\frac{4 M^{2} x_{\mathrm{Bj}}^{2} M_{\mathrm{B}, \mathrm{T}}^{2}}{Q^{4} z_{\mathrm{h}}^{2}}}} F_{T / L}=\left(1+\frac{\gamma^{2}}{2 x_{\mathrm{Bj}}}\right) F_{T / L}^{\mathrm{tot}} .
$$

Substituting eq. (2.24) for $z_{\mathrm{N}}$ gives the factor in eq. (2.37). Thus, the normalization of $F_{T / L}$ needs to be redefined as in eq. (2.38) in order to get the integration/sum rule in eq. (2.40) and [20, eqs. (2.18)-(2.21)].

\section{E Rapidity}

It is often useful to express results in terms of rapidity instead of $z_{\mathrm{N}}$ or $z_{\mathrm{h}}$. In the Breit frame,

$$
y_{P, \mathrm{~b}} \equiv \ln \left(\frac{Q}{x_{\mathrm{N}} M}\right), \quad y_{\mathrm{B}, \mathrm{b}} \equiv \ln \left(\frac{M_{\mathrm{B}, \mathrm{T}}}{z_{\mathrm{N}} Q}\right) .
$$

The boost invariant rapidity difference is

$$
\Delta y \equiv y_{P, \mathrm{~b}}-y_{\mathrm{B}, \mathrm{b}}=\ln \left(\frac{z_{\mathrm{N}} Q^{2}}{x_{\mathrm{N}} M M_{\mathrm{B}, \mathrm{T}}}\right) .
$$


If $x_{\mathrm{N}} \approx z_{\mathrm{N}}$ and $M_{\mathrm{B}, \mathrm{T}} \approx M$, then the produced hadron rapidity is approximately the negative of the proton rapidity. For fixed $z_{\mathrm{N}} / x_{\mathrm{N}}$, fixed $M_{\mathrm{B}, \mathrm{T}}$ and large $Q$

$$
e^{\Delta y}=O\left(\frac{Q^{2}}{m^{2}}\right), \quad e^{-\Delta y}=O\left(\frac{m^{2}}{Q^{2}}\right) .
$$

$z_{\mathrm{h}}$ in terms of $y_{\mathrm{B}, \mathrm{b}}$ is $[31]$

$$
z_{\mathrm{h}}=\frac{x_{\mathrm{N}} M_{\mathrm{B}, \mathrm{T}} M}{Q^{2}-x_{\mathrm{N}}^{2} M^{2}}\left(e^{\Delta y}+e^{-\Delta y}\right) \approx \frac{x_{\mathrm{Bj}} M_{\mathrm{B}, \mathrm{T}} M}{Q^{2}} e^{\Delta y} .
$$

In terms of $z_{\mathrm{h}}$, the rapidity of the hadron in the Breit frame is double valued:

$$
y_{\mathrm{B}, \mathrm{b}}^{ \pm}=\ln \left[\frac{Q z_{\mathrm{h}}\left(Q^{2}-x_{\mathrm{N}}^{2} M_{p}^{2}\right)}{2 x_{\mathrm{N}}^{2} M^{2} M_{\mathrm{B}, \mathrm{T}}} \pm \frac{Q}{x_{\mathrm{N}} M} \sqrt{\frac{z_{\mathrm{h}}^{2}\left(Q^{2}-x_{\mathrm{N}}^{2} M^{2}\right)^{2}}{4 x_{\mathrm{N}}^{2} M^{2} M_{\mathrm{B}, \mathrm{T}}^{2}}-1}\right] \approx \ln \left(\frac{M_{\mathrm{B}, \mathrm{T}}}{z_{\mathrm{h}} Q}\right) .
$$

The "+" solution corresponds to a hadron with large rapidity in the direction of $P$, while the "-" solution corresponds to a rapidity in the opposite direction, and thus is more consistent with current region factorization. The approximation after the " $\approx$ " corresponds to the $m^{2} / Q^{2} \rightarrow 0$ limit of the "-" solution.

Expressing the plus and minus components in eq. (4.1) in terms of rapidity,

$$
y_{\mathrm{i}}^{\mathrm{b}}=\frac{1}{2} \ln \left(\left|\frac{Q^{2}}{\hat{x}_{\mathrm{N}}^{2}\left(k_{\mathrm{i}}^{2}+\mathbf{k}_{\mathrm{i}, \mathrm{T}}^{2}\right)}\right|\right), \quad y_{\mathrm{f}}^{\mathrm{b}}=\frac{1}{2} \ln \left(\left|\frac{\hat{z}_{\mathrm{N}}^{2} q_{\mathrm{T}}^{2}+\delta k_{\mathrm{T}}^{2}-2 \hat{z}_{\mathrm{N}} \mathbf{q} \mathrm{T} \cdot \delta \mathbf{k}_{\mathrm{T}}+k_{\mathrm{f}}^{2}}{\hat{z}_{\mathrm{N}}^{2} Q^{2}}\right|\right) .
$$

Then, values of $\hat{z}_{\mathrm{N}}, \hat{x}_{\mathrm{N}}, k_{\mathrm{i}}, k_{\mathrm{f}}, \mathbf{k}_{\mathrm{i}, \mathrm{T}}, \mathbf{k}_{\mathrm{f}, \mathrm{T}}$ can be mapped, along with values of $R_{0}-R_{3}$, to regions of a $q_{\mathrm{T}}$ versus rapidity map like figure 8. If $\hat{z}_{\mathrm{N}} q_{\mathrm{T}}=O(Q)$, then $y_{\mathrm{f}}^{\mathrm{b}} \approx \ln \left(\frac{q_{\mathrm{T}}}{Q}\right) \approx 0$, while if $\hat{z}_{\mathrm{N}} q_{\mathrm{T}}=O(m)$, then $y_{\mathrm{f}}^{\mathrm{b}} \approx \ln \left(\frac{m}{Q}\right)$. In the handbag configuration, wherein all partonic transverse momenta are zero, the parton four-momenta may be written,

$$
k_{\mathrm{i}}=\left(\frac{\sqrt{-k_{\mathrm{i}}^{2}}}{\sqrt{2}} e^{y_{\mathrm{i}}^{\mathrm{b}}},-\frac{\sqrt{-k_{\mathrm{i}}^{2}}}{\sqrt{2}} e^{-y_{\mathrm{i}}^{\mathrm{b}}}, \mathbf{0}_{\mathrm{T}}\right), \quad k_{\mathrm{f}}=\left(\frac{\sqrt{k_{\mathrm{f}}^{2}}}{\sqrt{2}} e^{y_{\mathrm{f}}^{\mathrm{b}}}, \frac{\sqrt{k_{\mathrm{f}}^{2}}}{\sqrt{2}} e^{-y_{\mathrm{f}}^{\mathrm{b}}}, \mathbf{0}_{\mathrm{T}}\right) .
$$

Since $k_{\mathrm{i}}^{+} \approx-q_{\mathrm{b}}^{+}=Q / \sqrt{2}$ and $k_{\mathrm{f}}^{+} \approx q_{\mathrm{b}}^{-}=Q / \sqrt{2}$ in the handbag configuration, then $y_{\mathrm{i}}^{\mathrm{b}} \approx-y_{\mathrm{f}}^{\mathrm{b}}=O(\ln (Q / m))$. Therefore, partons in the handbag configuration are centered roughly on $y \approx 0$ in the Breit frame.

Note also that if $x_{\mathrm{N}}$ and $z_{\mathrm{N}}$ are small, then according to eq. (E.1) both the target and produced hadrons will tend to be skewed toward larger rapidities in the Breit frame. Therefore, hadrons measured in the final state will tend to be at larger rapidities than the corresponding handbag-configuration partons.

Finally we write down the formula for $R_{1}$ [31] in eq. (4.15), in terms of rapidity,

$$
R_{1}=\frac{M_{\mathrm{B}, \mathrm{T}} M_{\mathrm{f}, \mathrm{b}, \mathrm{T}}\left(e^{y_{\mathrm{B}, \mathrm{b}}-y_{\mathrm{f}}^{\mathrm{b}}}+e^{y_{\mathrm{f}}^{\mathrm{b}}-y_{\mathrm{B}, \mathrm{b}}}\right)-2 z_{\mathrm{N}} \hat{z}_{\mathrm{N}} q_{\mathrm{T}}^{2}+2 z_{\mathrm{N}} \mathbf{q}_{\mathrm{T}} \cdot \delta \mathbf{k}_{\mathrm{T}}}{M_{\mathrm{B}, \mathrm{T}} M_{\mathrm{i}, \mathrm{b}, \mathrm{T}}\left(e^{y_{\mathrm{i}}^{\mathrm{b}}-y_{\mathrm{B}, \mathrm{b}}}-e^{y_{\mathrm{B}, \mathrm{b}}-y_{\mathrm{i}}^{\mathrm{b}}}\right)+2 z_{\mathrm{N}} \mathbf{q}_{\mathrm{T}} \cdot \mathbf{k}_{\mathrm{i}, \mathrm{T}}},
$$

where $M_{\mathrm{i}, \mathrm{b}, \mathrm{T}}=\sqrt{\left|k_{\mathrm{i}}^{2}+\mathbf{k}_{\mathrm{i}, \mathrm{T}}^{2}\right|}$ and $M_{\mathrm{f}, \mathrm{b}, \mathrm{T}}=\sqrt{k_{\mathrm{f}}^{2}+\mathbf{k}_{\mathrm{f}, \mathrm{T}}^{2}}$. 
Open Access. This article is distributed under the terms of the Creative Commons Attribution License (CC-BY 4.0), which permits any use, distribution and reproduction in any medium, provided the original author(s) and source are credited.

\section{References}

[1] J. Dudek et al., Physics opportunities with the 12 GeV upgrade at Jefferson lab, Eur. Phys. J. A 48 (2012) 187 [arXiv: 1208.1244] [INSPIRE].

[2] A. Accardi et al., Electron ion collider: the next QCD frontier, Eur. Phys. J. A 52 (2016) 268 [arXiv: 1212.1701] [INSPIRE].

[3] E.-C. Aschenauer et al., Pre-town meeting on spin physics at an electron-ion collider, Eur. Phys. J. A 53 (2017) 71 [arXiv:1410.8831] [InSPIRE].

[4] H. Avakian, Studies of the 3D structure of the proton at Jlab, Phys. Part. Nucl. 45 (2014) 312 [INSPIRE].

[5] E.-C. Aschenauer et al., The RHIC cold QCD plan for 2017 to 2023: a portal to the EIC, arXiv: 1602.03922 [INSPIRE].

[6] H. Avakian, A. Bressan and M. Contalbrigo, Experimental results on TMDs, Eur. Phys. J. A 52 (2016) 150 [Erratum ibid. A 52 (2016) 165] [InSPIRE].

[7] L. Trentadue and G. Veneziano, Fracture functions: an improved description of inclusive hard processes in QCD, Phys. Lett. B 323 (1994) 201 [INSPIRE].

[8] D. Graudenz, One particle inclusive processes in deeply inelastic lepton-nucleon scattering, Nucl. Phys. B 432 (1994) 351 [hep-ph/9406274] [InSPIRE].

[9] T. Muta, Foundations of quantum chromodynamics: an introduction to perturbative methods in gauge theories, World Scientific, Singapore (1987).

[10] W. Greiner, S. Schramm and E. Stein, Quantum chromodynamics, Springer, Germany (2002).

[11] J.C. Collins, Foundations of perturbative QCD, Cambridge University Press, Cambridge U.K. (2011).

[12] N. Sato, T. Rogers and S. Gordon, Sidis region analysis tool, https://sidis.herokuapp.com.

[13] R.-b. Meng, F.I. Olness and D.E. Soper, Semiinclusive deeply inelastic scattering at electron-proton colliders, Nucl. Phys. B 371 (1992) 79 [INSPIRE].

[14] J. Levelt and P.J. Mulders, Quark correlation functions in deep inelastic semiinclusive processes, Phys. Rev. D 49 (1994) 96 [hep-ph/9304232] [INSPIRE].

[15] R. Meng, F.I. Olness and D.E. Soper, Semiinclusive deeply inelastic scattering at small $q_{T}$, Phys. Rev. D 54 (1996) 1919 [hep-ph/9511311] [InSPIRE].

[16] P.J. Mulders and R.D. Tangerman, The complete tree level result up to order $1 / Q$ for polarized deep inelastic leptoproduction, Nucl. Phys. B 461 (1996) 197 [Erratum ibid. B 484 (1997) 538] [hep-ph/9510301] [INSPIRE].

[17] P.M. Nadolsky, D.R. Stump and C.P. Yuan, Semiinclusive hadron production at HERA: the effect of QCD gluon resummation, Phys. Rev. D 61 (2000) 014003 [Erratum ibid. D 64 (2001) 059903] [hep-ph/9906280] [INSPIRE].

[18] P.J. Mulders, unpublished collected notes, http://www.nat.vu.nl/ mulders/correlations-0new.pdf. 
[19] M. Anselmino et al., The role of Cahn and Sivers effects in deep inelastic scattering, Phys. Rev. D 71 (2005) 074006 [hep-ph/0501196] [INSPIRE].

[20] A. Bacchetta et al., Semi-inclusive deep inelastic scattering at small transverse momentum, JHEP 02 (2007) 093 [hep-ph/0611265] [INSPIRE].

[21] A. Bacchetta, D. Boer, M. Diehl and P.J. Mulders, Matches and mismatches in the descriptions of semi-inclusive processes at low and high transverse momentum, JHEP 08 (2008) 023 [arXiv: 0803.0227] [INSPIRE].

[22] M. Anselmino et al., General helicity formalism for polarized semi-inclusive deep inelastic scattering, Phys. Rev. D 83 (2011) 114019 [arXiv:1101.1011] [INSPIRE].

[23] E. Moffat et al., What does kinematical target mass sensitivity in DIS reveal about hadron structure?, Phys. Rev. D 99 (2019) 096008 [arXiv:1901.09016] [INSPIRE].

[24] A. Bacchetta, U. D'Alesio, M. Diehl and C.A. Miller, Single-spin asymmetries: the Trento conventions, Phys. Rev. D 70 (2004) 117504 [hep-ph/0410050] [INSPIRE].

[25] J.V. Guerrero et al., Hadron mass corrections in semi-inclusive deep-inelastic scattering, JHEP 09 (2015) 169 [arXiv: 1505.02739] [INSPIRE].

[26] E. Byckling and K. Kajantie, Particle kinematics, University of Jyvaskyla, Jyvaskyla, Finland (1971).

[27] HERMES collaboration, Multiplicities of charged pions and kaons from semi-inclusive deep-inelastic scattering by the proton and the deuteron, Phys. Rev. D 87 (2013) 074029 [arXiv: 1212.5407] [INSPIRE].

[28] COMPASS collaboration, Transverse-momentum-dependent multiplicities of charged hadrons in muon-deuteron deep inelastic scattering, Phys. Rev. D 97 (2018) 032006 [arXiv: 1709.07374] [INSPIRE].

[29] A. Accardi, D.P. Anderle and F. Ringer, Interplay of threshold resummation and hadron mass corrections in deep inelastic processes, Phys. Rev. D 91 (2015) 034008 [arXiv: 1411.3649] [INSPIRE].

[30] J.C. Collins, D.E. Soper and G.F. Sterman, Factorization of hard processes in QCD, Adv. Ser. Direct. High Energy Phys. 5 (1989) 1 [hep-ph/0409313] [INSPIRE].

[31] M. Boglione et al., Kinematics of current region fragmentation in semi-inclusive deeply inelastic scattering, Phys. Lett. B 766 (2017) 245 [arXiv:1611.10329] [INSPIRE].

[32] J.O. Gonzalez-Hernandez, T.C. Rogers, N. Sato and B. Wang, Challenges with large transverse momentum in semi-inclusive deeply inelastic scattering, Phys. Rev. D 98 (2018) 114005 [arXiv: 1808.04396] [INSPIRE].

[33] Y. Koike, J. Nagashima and W. Vogelsang, Resummation for polarized semi-inclusive deep-inelastic scattering at small transverse momentum, Nucl. Phys. B 744 (2006) 59 [hep-ph/0602188] [INSPIRE].

[34] M. Anselmino, M. Boglione, A. Prokudin and C. Turk, Semi-inclusive deep inelastic scattering processes from small to large $p_{T}$, Eur. Phys. J. A 31 (2007) 373 [hep-ph/0606286] [INSPIRE].

[35] M. Anselmino et al., Unpolarised transverse momentum dependent distribution and fragmentation functions from SIDIS multiplicities, JHEP 04 (2014) 005 [arXiv:1312.6261] [INSPIRE]. 
[36] A. Signori, A. Bacchetta, M. Radici and G. Schnell, Investigations into the flavor dependence of partonic transverse momentum, JHEP 11 (2013) 194 [arXiv:1309.3507] [INSPIRE].

[37] P. Sun and F. Yuan, Energy evolution for the Sivers asymmetries in hard processes, Phys. Rev. D 88 (2013) 034016 [arXiv: 1304.5037] [INSPIRE].

[38] P. Sun and F. Yuan, Transverse momentum dependent evolution: Matching semi-inclusive deep inelastic scattering processes to Drell-Yan and $W / Z$ boson production, Phys. Rev. D 88 (2013) 114012 [arXiv:1308.5003] [INSPIRE].

[39] A. Bacchetta, F. Delcarro, C. Pisano, M. Radici and A. Signori, Extraction of partonic transverse momentum distributions from semi-inclusive deep-inelastic scattering, Drell-Yan and Z-boson production, JHEP 06 (2017) 081 [Erratum ibid. 06 (2019) 051] [arXiv: 1703.10157] [INSPIRE].

[40] M. Anselmino et al., Study of the sign change of the Sivers function from STAR Collaboration W/Z production data, JHEP 04 (2017) 046 [arXiv: 1612.06413] [INSPIRE].

[41] M. Anselmino et al., Collins functions for pions from SIDIS and new $e^{+} e^{-}$data: a first glance at their transverse momentum dependence, Phys. Rev. D 92 (2015) 114023 [arXiv: 1510.05389] [INSPIRE].

[42] A. Bacchetta et al., Effects of TMD evolution and partonic flavor on $e^{+} e^{-}$annihilation into hadrons, JHEP 11 (2015) 076 [arXiv: 1508.00402] [INSPIRE].

[43] I. Scimemi and A. Vladimirov, Analysis of vector boson production within TMD factorization, Eur. Phys. J. C 78 (2018) 89 [arXiv:1706.01473] [INSPIRE].

[44] Z.-B. Kang, A. Prokudin, F. Ringer and F. Yuan, Collins azimuthal asymmetries of hadron production inside jets, Phys. Lett. B 774 (2017) 635 [arXiv:1707.00913] [INSPIRE].

[45] Z.-B. Kang, A. Prokudin, P. Sun and F. Yuan, Nucleon tensor charge from Collins azimuthal asymmetry measurements, Phys. Rev. D 91 (2015) 071501 [arXiv:1410.4877] [INSPIRE].

[46] F. Landry, R. Brock, P.M. Nadolsky and C.P. Yuan, Tevatron run-1 Z boson data and Collins-Soper-Sterman resummation formalism, Phys. Rev. D 67 (2003) 073016 [hep-ph/0212159] [INSPIRE].

[47] P. Sun, J. Isaacson, C.P. Yuan and F. Yuan, Resummation of high order corrections in Higgs boson plus jet production at the LHC, Phys. Lett. B 769 (2017) 57 [arXiv:1602.08133] [INSPIRE].

[48] P. Sun, B.-W. Xiao and F. Yuan, Gluon distribution functions and Higgs boson production at moderate transverse momentum, Phys. Rev. D 84 (2011) 094005 [arXiv:1109.1354] [INSPIRE].

[49] M.G. Echevarria, T. Kasemets, P.J. Mulders and C. Pisano, QCD evolution of (un)polarized gluon TMDPDFs and the Higgs $q_{T}$-distribution, JHEP 07 (2015) 158 [Erratum ibid. 05 (2017) 073] [arXiv: 1502.05354] [INSPIRE].

[50] M. Guzzi, P.M. Nadolsky and B. Wang, Nonperturbative contributions to a resummed leptonic angular distribution in inclusive neutral vector boson production, Phys. Rev. D 90 (2014) 014030 [arXiv: 1309.1393] [INSPIRE].

[51] M.G. Echevarria, I. Scimemi and A. Vladimirov, Unpolarized transverse momentum dependent parton distribution and fragmentation functions at next-to-next-to-leading order, JHEP 09 (2016) 004 [arXiv: 1604.07869] [INSPIRE]. 
[52] M.G. Echevarria, A. Idilbi, Z.-B. Kang and I. Vitev, QCD evolution of the Sivers asymmetry, Phys. Rev. D 89 (2014) 074013 [arXiv: 1401.5078] [InSPIRE].

[53] M. Boglione, U. D'Alesio, C. Flore and J.O. Gonzalez-Hernandez, Assessing signals of TMD physics in SIDIS azimuthal asymmetries and in the extraction of the Sivers function, JHEP 07 (2018) 148 [arXiv:1806.10645] [INSPIRE].

[54] M. Boglione, J.O. Gonzalez-Hernandez and R. Taghavi, Transverse parton momenta in single inclusive hadron production in $e^{+} e^{-}$annihilation processes, Phys. Lett. B 772 (2017) 78 [arXiv: 1704.08882] [INSPIRE].

[55] Z.-B. Kang, A. Prokudin, P. Sun and F. Yuan, Extraction of quark transversity distribution and collins fragmentation functions with QCD evolution, Phys. Rev. D 93 (2016) 014009 [arXiv: 1505.05589] [INSPIRE].

[56] H.-W. Lin, W. Melnitchouk, A. Prokudin, N. Sato and H. Shows, First Monte Carlo global analysis of nucleon transversity with lattice QCD constraints, Phys. Rev. Lett. 120 (2018) 152502 [arXiv: 1710.09858] [INSPIRE].

[57] Z. Ye et al., Unveiling the nucleon tensor charge at Jefferson lab: a study of the SoLID case, Phys. Lett. B 767 (2017) 91 [arXiv:1609.02449] [INSPIRE].

[58] A. Bacchetta et al., Difficulties in the description of Drell-Yan processes at moderate invariant mass and high transverse momentum, Phys. Rev. D 100 (2019) 014018 [arXiv: 1901.06916] [INSPIRE].

[59] S. Bastami et al., Semi-inclusive deep inelastic scattering in Wandzura-Wilczek-type approximation, JHEP 06 (2019) 007 [arXiv:1807.10606] [INSPIRE].

[60] V. Bertone, I. Scimemi and A. Vladimirov, Extraction of unpolarized quark transverse momentum dependent parton distributions from Drell-Yan/Z-boson production, JHEP 06 (2019) 028 [arXiv: 1902.08474] [INSPIRE].

[61] M. Diehl and S. Sapeta, On the analysis of lepton scattering on longitudinally or transversely polarized protons, Eur. Phys. J. C 41 (2005) 515 [hep-ph/0503023] [INSPIRE].

[62] X.-d. Ji, J.-P. Ma and F. Yuan, QCD factorization for spin-dependent cross sections in DIS and Drell-Yan processes at low transverse momentum, Phys. Lett. B 597 (2004) 299 [hep-ph/0405085] [INSPIRE].

[63] A. Idilbi, X.-d. Ji, J.-P. Ma and F. Yuan, Collins-Soper equation for the energy evolution of transverse-momentum and spin dependent parton distributions, Phys. Rev. D 70 (2004) 074021 [hep-ph/0406302] [INSPIRE].

[64] X.-d. Ji, J.-p. Ma and F. Yuan, QCD factorization for semi-inclusive deep-inelastic scattering at low transverse momentum, Phys. Rev. D 71 (2005) 034005 [hep-ph/0404183] [INSPIRE]. 